\title{
Systematic drug screening reveals specific vulnerabilities and co-resistance patterns in endocrine-resistant breast cancer
}

Sara Kangaspeska ${ }^{1,2^{*}+}$, Susanne Hultsch ${ }^{1 \dagger}$, Alok Jaiswal ${ }^{1}$, Henrik Edgren ${ }^{1,3}$, John-Patrick Mpindi ${ }^{1}$, Samuli Eldfors ${ }^{1}$, Oscar Brück', Tero Aittokallio ${ }^{1}$ and Olli Kallioniemi ${ }^{1,4}$

\begin{abstract}
Background: The estrogen receptor (ER) inhibitor tamoxifen reduces breast cancer mortality by $31 \%$ and has served as the standard treatment for ER-positive breast cancers for decades. However, $50 \%$ of advanced ER-positive cancers display de novo resistance to tamoxifen, and acquired resistance evolves in $40 \%$ of patients who initially respond. Mechanisms underlying resistance development remain poorly understood and new therapeutic opportunities are urgently needed. Here, we report the generation and characterization of seven tamoxifen-resistant breast cancer cell lines from four parental strains.

Methods: Using high throughput drug sensitivity and resistance testing (DSRT) with 279 approved and investigational oncology drugs, exome-sequencing and network analysis, we for the first time, systematically determine the drug response profiles specific to tamoxifen resistance.

Results: We discovered emerging vulnerabilities towards specific drugs, such as ERK1/2-, proteasome- and $\mathrm{BCL}$-family inhibitors as the cells became tamoxifen-resistant. Co-resistance to other drugs such as the survivin inhibitor YM155 and the chemotherapeutic agent paclitaxel also occurred.

Conclusion: This study indicates that multiple molecular mechanisms dictate endocrine resistance, resulting in unexpected vulnerabilities to initially ineffective drugs, as well as in emerging co-resistances. Thus, combatting drug-resistant tumors will require patient-tailored strategies in order to identify new drug vulnerabilities, and to understand the associated co-resistance patterns.
\end{abstract}

Keywords: Tamoxifen resistance, Breast cancer, High-throughput drug testing, Exome-sequencing, Drug resistance

\section{Background}

Breast cancer is the most common cancer in women worldwide. Two-thirds of breast tumors express ER that drives proliferation of mammary epithelial cells and thereby contributes to the etiology and progression of the disease. Consequently, antagonists that directly block ER function or drugs that lower the amounts of the natural ligand of ER, estradiol, have been utilized in breast cancer treatment for decades [1]. For over 40 years,

\footnotetext{
* Correspondence: sara.kangaspeska@helsinki.fi

${ }^{\dagger}$ Equal contributors

${ }^{1}$ Institute for Molecular Medicine Finland (FIMM), Biomedicum 2U,

Tukholmankatu 8, 00290 Helsinki, Finland

2Present address: Helsinki Innovation Services, Tukholmankatu 8 A, 00290

Helsinki, Finland

Full list of author information is available at the end of the article
}

tamoxifen, a selective ER antagonist, has been the backbone in treating ER-positive breast cancers. Despite of being effective in decreasing mortality, de novo or acquired resistance frequently occurs [2]. Some of the mechanisms leading to resistance have been revealed, including mutations in the gene encoding ER [3-5], altered expression patterns of ER or its cofactors [6, 7], and crosstalk between ER and growth factor receptor cascades such as the EGFR/ERK1/2-pathway [8]. Consequently, inhibition of ERK1/2 has been reported to restore antiestrogen sensitivity. For example, a study with the MEK inhibitor PD098059, a compound that reduces the phosphorylation and activation of ERK1/2, was shown to inhibit the growth of tamoxifen-resistant cell lines and to restore their sensitivity to therapy $[9,10]$. 
However, ERK1/2 inhibition has proven efficacy primarily against cells with resistance-provoked overexpression or activation of HER2 [9]. On the other hand, recent findings suggest that proteasome inhibition might offer a new avenue for overcoming endocrine resistance [11, 12]. Bortezomib, a proteasome inhibitor, has been investigated as a combination therapy in conjunction with endocrine treatment in a phase II study [13].

Whilst shRNA- or cDNA-based functional screens $[14,15]$ and candidate gene [16-19], or drug [9, 20-23] approaches have been used to study the development and reversal of endocrine resistance, the exact molecular mechanisms remain unknown, and large-scale studies on cells treated long-term with tamoxifen are lacking. Moreover, efforts to find new treatment regimes for overcoming drug resistance have been largely based on a few selected drug candidates, and have only proven to be effective in a fraction of the cases [1]. Development of primary drug resistance can make the cancer cells susceptible for novel vulnerabilities, hence leading to additional therapeutic opportunities. However, secondary resistances towards other drugs may also arise. Resistance to chemotherapeutics has been linked with estrogen receptor positive breast cancer [24], but systematic studies on tamoxifen resistance associated coresistances have not been conducted. Therefore, systematic, large-scale studies to characterize the drug sensitivity profiles of tamoxifen-resistant breast cancer are warranted to reveal new drug vulnerabilities as well as co-resistance patterns in drug-resistant cells.

Here, we report the development and characterization of a panel of seven long-term tamoxifen-treated breast cancer cell lines from four parental strains. Using these resistant cell line models and their isogenic parental counterparts, we, for the first time, performed systematic high throughput drug sensitivity and resistance testing with 279 approved and investigational oncology drugs to reveal potential new drug vulnerabilities and to identify coresistance patterns acquired with tamoxifen resistance. We further conducted exome-sequencing on each of the isogenic parental-resistant cell line pair to identify point mutations and copy number variations that may contribute to drug resistance. Through integrated network analyses, we uncovered cell- and clone-specific molecular and functional patterns of endocrine resistance, highlighting the underlying molecular diversity, and pointing to several distinct therapeutic opportunities to circumvent it. However, no systematic drug screens with hundreds of oncology compounds on acquired tamoxifen resistance have been conducted.

\section{Methods}

\section{Cell culture}

Human breast cancer cell lines MCF-7 (HTB-22, ATCC), T-47D (HTB-133, ATCC), ZR-75-1 (CRL-1500,
ATCC) and BT-474 (HTB-20, ATCC) were obtained from the American Type Culture Collection. The cells were grown in DMEM with L-Glutamine (MCF-7 and BT-474, PAN Biotech, Aidenbach, Germany) or RPMI1640 with L-Glutamine (ZR-75-1 and T-47D, PAN Biotech) supplemented with $10 \%$ FCS (Gibco, Life Technologies, Carlsbad, CA) and $1 \%$ penicillin/streptomycin (Gibco). Culture media for T-47D, MCF-7 and BT-474 additionally contained $0,1 \%$ bovine insulin (Sigma. St. Louis, MO). The tamoxifen-resistant cell lines (MCF-7 Tam1, T-47D Tam1 \& Tam2, ZR-75-1 Tam1 \& Tam2, BT-474 Tam1 \& Tam2) were derived from the parental cell lines by continuous exposure to 4OH-tamoxifen (Sigma, $1 \mu \mathrm{M}$ in ethanol) for 8-12 months. Culture media was replaced every $2-3$ days. All cells were incubated at $37{ }^{\circ} \mathrm{C}$ with $5 \% \mathrm{CO}_{2}$ and passaged when ca $80 \%$ confluent. The approximate doubling times of the cells were as follows: parental MCF-7, T47D, ZR-75-1 and BT-474: 1-3 days. Resistant MCF-7 Tam1, T-47D Tam1 and Tam2: 1-2 weeks, ZR-75-1 Tam1 and Tam2: > 1 week, BT-474 Tam1 and Tam2: 2 weeks. The cells were free of mycoplasma and verified for their authenticity (Technology Centre, Institute for Molecular Medicine Finland, Helsinki, Finland).

\section{Characterization of tamoxifen-resistant cell lines}

For viability measurements cells were seeded in 384-well culture plates with increasing tamoxifen concentrations $(0-1,8 \mu \mathrm{M})$. After $120 \mathrm{~h}$ cell viability was evaluated by CellTiter-Glo Cell Viability Assay (Promega, Fitchburg, WI) with the PHERAstar plate reader (Agilent Technologies Santa Clara, CA). To measure the active DNA synthesis cell proliferation assays with Click-iT ${ }^{\circ}$ EdU Alexa Fluor 488 Flow Cytometry Assay Kit were performed according to manufacturer's protocol (Life Technologies) with following minor modifications: Cells were plated on $10 \mathrm{~cm}$ plates and cultured with and without $1 \mu \mathrm{M}$ tamoxifen until approximately $50 \%$ confluent. Parental cells and their tamoxifen-resistant derivatives were then pulsed with $10 \mu \mathrm{M}$ of EdU Alexa Fluor ${ }^{\circ} 488$ for $4 \mathrm{~h}$ (T-47D and MCF-7) or for $28 \mathrm{~h}$ (BT-474 and ZR-75-1). Cells were permeabilized with saponin-based permeabilization and wash reagent (MCF-7, T-47D, BT474 ) or with $0,1 \%$ TritonX-100-PBS (ZR-75-1) for $10 \mathrm{~min}$. Additionally, DNA content staining was performed using FxCycle ${ }^{\mathrm{Tt}}$ Far Red and the cell suspension treated with RibonucleaseA. Flow cytometry was carried out and results analyzed using Accuri C6 flow cytometer and associated software (BD Biosciences, Franklin Lakes, $\mathrm{NJ})$. To measure estrogen-responsivity of the parental cells and their tamoxifen-resistant derivatives, the cells were grown on 6-well plates in hormone-deprived culture medium for $72 \mathrm{~h}$ (phenol red-free DMEM or RPMI, PAN Biotech), supplemented with 2,5 \% dextran- 
charcoal-treated (Sigma-Aldrich) FCS and other additives (see above). $17 \beta$-estradiol (Sigma, $10^{-8} \mathrm{M}$ in ethanol) was then added back to the cells for 4,8 or $24 \mathrm{~h}$ and RNA isolated with Total RNA Purification kit (Norgen, Thorold, ON). $4 \mu \mathrm{g}$ of total RNA were reverse transcribed with the High-Capacity cDNA Reverse transcription kit (Applied Biosystems, Thermo Scientific, Waltham, MA) as instructed. Quantitative-PCR was then performed on the LightCycler 480 system (Roche, Penzberg, Germany) using the DyNAmo colour flash SYBRGreen PCR kit (Thermo Scientific) with equal amounts of cDNA. The optimal internal reference gene was determined out of a pool of 16 different housekeeping genes for each parental-resistant cell line pair (18S for MCF-7 s, PPIA for T-47Ds and B2M for ZR-75-1 s and BT-474 s). Primer sequences can be found in Additional file 1. All experiments were done in triplicates. For Western Blotting cells were grown on $10 \mathrm{~cm}$ dishes, and lyzed in Laemmli buffer. Immunoblotting was done as previously described [25]. The used antibodies were as follows: ER $\alpha$ (Abcam, Cambridge, UK, ab16660), $\beta$ actin (Sigma-Aldrich, A1978), EGFR (Cell Signaling Technologies, CST4267), phospho-EGFR (CST3777), ERK1/2 (CST9107), phospho-ERK1/2 (CST4370). To test the effects the ERK inhibitor VX-11E and the MEK inhibitor selumintinib the cells were cultured either in their default culture media with no additional drug, or with increasing concentrations of VX-11E (50nM, $100 \mathrm{nM}$ and $250 \mathrm{nM}$ ), or with $100 \mathrm{nM}$ VX-11E in combination with $1 \mu \mathrm{M}$ selumetinib. The cells were then harvested and Western blotting with the above-mentioned antibodies performed.

\section{Genomic profiling by exome-sequencing}

Genomic DNA was isolated from the parental and tamoxifen-resistant cells using the DNeasy Blood \& Tissue kit (Qiagen, Venlo, Netherlands and Hilden, Germany). Exome-capture was done on $3 \mu \mathrm{g}$ DNA with the NimbleGen SeqCap EZ Human Exome v2.0 kit (Roche NimbleGen) and paired-end sequencing performed on Illumina HiSeq platform. Point mutations were detected as previously described [26], using the parental cell lines as controls. Briefly, point mutations specific to resistant samples were called with VarScan2 somatic [27], with the following parameters: strand-filter 1 , min-coverage-normal 8 , min-coverage-tumor 6 , somatic- $p$-value 1 , normal-purity 1 , min-var-freq 0,05 . Parental cell line was used as the normal control. Mutation calling was done within the exome capture target regions of the NimbleGen SeqCap EZ v2 capture kit and the flanking 500 bps. Mutations were annotated with SnpEff [28] using the Ensembl v66 annotation database. To filter out misclassified germline variants, common population variants included in dbSNP version 135 were removed. Remaining non-synonymous mutations were visually validated using the Integrated Genomics Viewer (Broad Institute). Mutations with $p<0,05$ and resistant variant frequency $>30 \%$ were deemed high confidence. Known false positive point mutations [29] were excluded. Exome-sequencing data was also analyzed using the sequence alignment and variant calling pipeline VCP. As input in the CNV analysis we used alignments in BAM format, as well as identified variants in all samples [30] (and unpublished). All exome sequencing capture kit target regions less than $76 \mathrm{bp}$ apart were merged with each other. An RPKM (reads per kilobase of target region length per million mapped reads) copy number value was calculated separately for every target region, followed by filtering out regions with sequencing coverage lower than 25x. Finally, relative $\log 2$ copy number ratios for sample (drug-resistant variant) divided by reference (parental cell line) were calculated and segmented using Circular Binary Segmentation [31]. Plots of copy number, segmentation and variant allele frequencies in capture target regions were visualized using R. Gene level copy number data for all human genes in Ensembl database v67 was calculated by assigning a gene the value of the CNV data segment that it overlapped. When a gene overlapped more than one segment, the gene was assigned a copy number value based on a modification of the extreme method option in GISTIC2 [32-34] as follows: the gene was given the lowest segment $\log 2$ value in case any overlapped segment had $\log 2$ ratio $<=-0,6$ and the highest segment value if any overlapped segment had $\log 2$ ratio $>=0,5$. If all segments the gene overlapped had $\log 2$ ratio $>-0,6$ and $<0,5$; the gene was assigned the median $\log 2$ ratio of all overlapped segments. Thresholds for copy number changes were determined based on samples (not published here) with known copy number differences, such as male vs female comparisons on chromosome $\mathrm{X}$, as well as trisomies observed in karyotyping of cells during routine diagnostics. Based on these, the limits were set at $-0,4$ (heterozygous deletion), $-1,2$ (homozygous deletion), $+0,5$ (gain) and $+1,3$ (amplification). Raw exome-sequencing data have been deposited in the NCBI Sequence Read Archive [SRP: SRP050366].

\section{Drug sensitivity and resistance testing (DSRT)}

DSRT with the FIMM FO2Baq library containing 279 approved and investigational oncology drugs was done as previously described [26] with minor modifications. Briefly, drugs were dissolved and plated in five different concentrations covering a 10 000-fold concentration range into the wells of 384-well plates. Optimized amounts of cells were then seeded into the wells in their normal growth media, i.e. parental cells in normal media and tamoxifen-resistant cells in media supplemented 
with $1 \mu \mathrm{M}$ 4-OH-tamoxifen. Thus, this set-up allows for measurement of permanent drug response changes corresponding to long-term tamoxifen treatment used in the clinical setting, and allows for any combinatorial drug responses to be observed. A further Cells were incubated at $37{ }^{\circ} \mathrm{C}$ for $72 \mathrm{~h}$ and viability measured by CellTiter-Glo Cell Viability Assay with the PHERAstar plate reader. Data were normalized to negative (DMSO only) as well as positive (100 $\mu \mathrm{mol} / \mathrm{l}$ benzethonium chloride) controls. The logistic dose-response curves were estimated using the Marquardt-Levenberg algorithm and implemented in the in-house bioinformatic pipeline Breeze. The dose-response curves were then employed to quantitatively profile drug responses, i.e. the Drug Sensitivity Score (DSS), which is based on the estimated logistic model parameters, and the DSS difference, which quantifies the differential drug response between tamoxifen-resistant and parental cells, as previously described $[26,35]$. We found that $|\mathrm{dDSS}|=5$ cutoff lies in the tail end of the distribution with $9.7 \%$ of values above the cutoff. With a two-tailed distribution (signed DSS), this would correspond to ca. $5 \%$ "hit rate", which we deemed as appropriate for such a drug discovery approach. Clustering of the DSS response differences across resistant/parental cell line pairs was performed using unsupervised hierarchical complete-linkage clustering, using Spearman and Euclidean distance measures of the drug and sample profiles, respectively, and visualized as a heat map [35]. In order to identify drugs that significantly change their efficacy as the cells gain resistance to tamoxifen, we performed rank product analysis [36] at false discovery rate of $5 \%(\mathrm{q}<0,05)$ by comparing drug response profiles in the parental cells against response profiles in the tamoxifen-resistant cells. The average DSS activity of a drug in all parental cell lines was plotted against the average DSS activity in the resistant clones. The drugs selected from the rank product analysis were considered as significant hits and displayed as colored dots. Luminal A or B subtype-specific drugs were identified (Additional file 2) based on the known subtypes of the parental cells [37].

\section{Construction of drug sensitivity and co-resistance networks}

To visualize drug sensitivity and resistance networks in each resistant/parental cell line pair, drugs with DSS difference $>5$ (sensitivity) or $<-5$ (co-resistance) were selected. For each drug, specific target molecules were defined using the KIBA (Kinase Inhibitor BioActivity) -score [38] as follows: First, drug target bioactivity (Ki, $\mathrm{Kd}$ and $\mathrm{IC}_{50}$ ) values were extracted from the ChEMBL database (https://www.ebi.ac.uk/chembl), and integrated KIBA-score was calculated for each drug-target pair. A low KIBA-score indicates a high binding affinity of the drug with the target. Amongst the set of targets for a drug, if the target with highest binding affinity had KIBA-score of $<0,1$, the specific target threshold was considered to be 50 -fold the lowest KIBA-value; otherwise KIBA-score $<3$ units was considered as the specific target threshold. Second, to capture the connection between genomic changes and drug-target genes of the most effective drugs in each resistant cell model compared to its parental cell line, network analysis and visualization was done on KIBA-scored specific targets genes of the sensitizing or desensitizing drugs using Ingenuity Pathway Analysis application (Ingenuity ${ }^{\circ}$ Systems, Qiagen). The IPA system is based on the Ingenuity Pathways Knowledge Base, which is a repository of $\mathrm{cu}-$ rated biological interactions, and functional annotations between proteins, genes, complexes extracted from scientific articles. The network consists of thousands of nodes and the edges represent experimentally observed cause-effect relationships that are related to direct physical interactions or regulatory interactions events like expression, transcription, activation and molecular modifications. Network edges are also associated with a direction of the causal effect, i.e. either activating or inhibiting [39]. Sensitizing and desensitizing drugs were considered separately. We required each resistant/parental cell line pair to have at least three effector drugs with a DSS difference $>5$ to enable network building based on their target genes, discarding the sensitivity networks for MCF-7 Tam1 and ZR-75-1 Tam1 as each of them had one effector drug only. The target molecule networks representing the most effective sensitizing or desensitizing drugs were merged, and then adjusted to keep the edges that were human-specific and high confidence. The resulting networks were then extended to upstream neighboring molecules to reveal connections with genes containing copy number variations or somatic mutations within the target networks. In the IPA framework, upstream molecules are defined as the genes that have been shown to affect the gene expression in some direct/indirect way [39]. The IPA network generation algorithm considers all the immediate upstream molecules separated by one degree from the nodes in the current network, where priority is given to those genes having maximum number of overlaps with the existing network. Furthermore, we merged the IPA canonical pathway for Estrogen Receptor Signaling (www.qiagen.com) with the networks utilizing the IPA overlay tool.

\section{Results}

\section{Development and characterization of long-term} tamoxifen-treated cell lines

To study the development of tamoxifen resistance across distinct molecular backgrounds, we established seven long-term tamoxifen-treated cell lines originating from 
four parental cell types; MCF-7, T-47D, ZR-75-1 and BT-474. We exposed these ER-positive, initially tamoxifen-responsive cells to continuous $1 \mu \mathrm{M} 4-\mathrm{OH}-$ tamoxifen treatment (hereafter tamoxifen) for 8 to 12 months (Fig. 1). A steady concentration of tamoxifen with clear inhibitory effect on ER-mediated transcription was chosen to mimic the exposure of patients to the drug in the clinic. In the course of the treatment, all cell cultures underwent cell death leaving a few founder cells that then recovered and repopulated the culture plates. Once stable, the cultures were molecularly characterized and subjected to pharmacogenomic profiling through drug sensitivity and resistance testing (DSRT) and exome-sequencing (Fig. 1). To confirm the resistant phenotypes, we assessed the viability of the cells upon increasing tamoxifen concentrations. All resistant cells showed increased tolerance towards tamoxifen compared to the parental cells, verifying the resistance development (Additional file 3A). For further characterization, we studied the cell cycle properties, functionality and levels of ER, and estrogen-responsivity of the long-term tamoxifentreated cells. Resistant cell clones exhibited altered cell cycle properties, with a higher fraction of cells in the G0/
G1 phase (Additional file 3B). All resistant cells had diminished ER target gene transcription, whereas the level of ER itself either increased or decreased depending on the clone (Additional file 4). Estradiol (E2) deprivation and subsequent addition of estradiol back to the cells revealed aberrant levels of ER target gene transcription, indicating that upon acquiring resistance to the ER antagonist tamoxifen, transcriptional regulation in response to the natural ligand of the receptor, estradiol, is disturbed.

\section{Acquired sensitivities and co-resistances upon development of tamoxifen resistance}

To uncover drug response profiles associated with longterm tamoxifen treatment mimicking the prolonged exposure to the drug used in the clinic, we conducted drug sensitivity and resistance testing (DSRT) [26] with 279 FDA/EMA-approved and investigational oncology drugs ranging from conventional chemotherapeutics to a variety of targeted drugs (Additional files 5 and 6). We quantified the overall drug responses for each compound in each cell line using drug sensitivity score (DSS) [35]. Targeted drugs generally had higher efficacy than the standard chemotherapeutics, as seen for example with

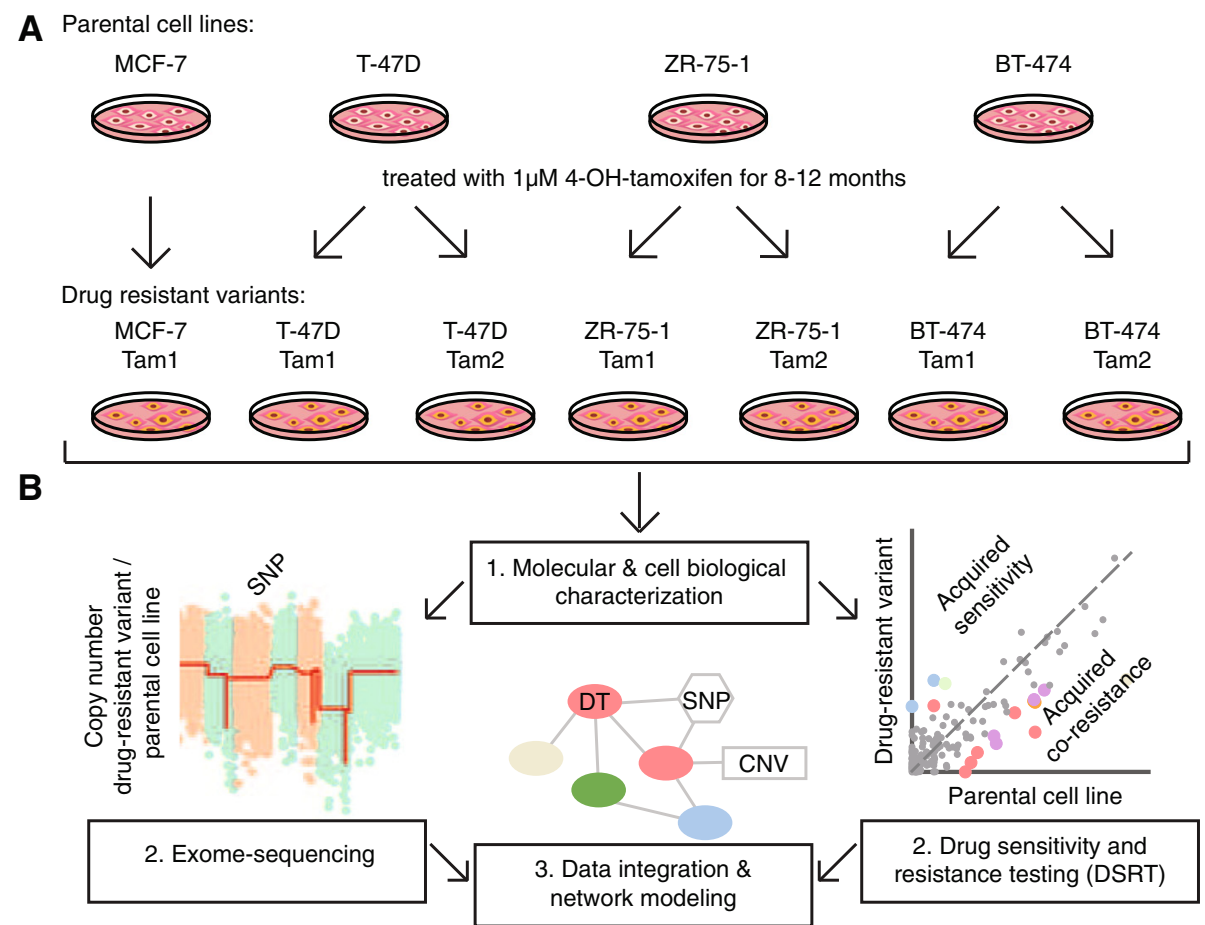

Fig. 1 Development and analyses of the parental breast cancer cell lines and their tamoxifen-resistant variants. a Schematic representation of the four parental cell lines (MCF-7, T-47D, ZR-75-1 and BT-474) and their seven tamoxifen-resistant variants (arrows) that were developed through continuous tamoxifen-treatment. b Followed by molecular and cell biological characterization (1), drug sensitivity and resistance testing and exome-sequencing to detect point mutations (single nucleotide polymorphisms, SNP) and copy number variations (CNV) (2), the data was then integrated and the pharmacogenomic relationships visualized through network modeling (3). DT= Drug target. To reveal drug responses and genetic changes associated with tamoxifen resistance, all analyses were performed by comparing each tamoxifen-resistant cell line to its parental control cell line 
the HER2/EGFR inhibitors lapatinib, afatinib and neratinib that were efficacious only against the BT-474 parental cells that express these receptors (Additional file 2A and B). We then calculated the DSS difference between the tamoxifen-resistant and their parental cells to reveal the cell line-specific drug sensitivity and resistance patterns associated with the resistance to tamoxifen. Interestingly, all tamoxifen-resistant cell lines displayed distinct drug response profiles, with a few overlapping responses (Fig. 2a). Overall, the drug responses of the resistant clones derived from the same parentals resembled each other more than those of the other resistant clones, and a few shared sensitivities arose, such as towards tyrosine kinase (gefinitib, ibrutinib) and cdk inhibitors (alvocidib, SNS-032) in the BT-474 clones. However, in T-47D and ZR-75-1, only a few common drug sensitivities and co-resistance patterns evolved between the two subclones, indicating clonal evolution (Fig. 2b).

\section{Resistant cells accumulate genetic alterations throughout their genomes}

To define the mutational landscape of the tamoxifenresistant cell clones and to identify potential genomic markers for drug sensitivity and resistance, we conducted exome-sequencing on each isogenic parentalresistant cell line pair. In order to identify resistancespecific point mutations and copy number variations, each tamoxifen-resistant cell line was compared to its parental cell line. Between 31,8 and 83,0 million uniquely mapping reads per sample were obtained with an average coverage of $43,7 x$ (Additional file 7 ). We found genetic changes scattered along the genomes of the tamoxifen-resistant cells (Additional files 8, 9 and 10). We identified approximately 250 to 350 nonsynonymous mutations per resistant cell line (Additional file 9), and for integration with copy number alterations, selected the high confidence ones. Interestingly, only a limited panel of mutated genes was shared between the two clones derived from the same parental cells including TIMM23 and RP11-368 J21.2.1 in ZR-75-1 Tam1 and Tam2, and TNS1, PTH2R and NHLRC2 in BT-474 Tam1 and Tam2. None of the point mutations were shared between T-47D Tam1 and Tam2 (Additional file 8). All resistant clones displayed marked copy number aberrations, which were primarily large deletions, with the exception of ZR-75-1 Tam1 that only harbored gains. Chromosomes $\mathrm{X}$ and seven were recurrently altered, with few larger deletions (such as a homo- and heterozygous deletion of $\mathrm{Xq}$ in MCF-7 Tam1 and heterozygous deletion of 7q in T-47D Tam1), as well as several smaller aberrations found in each resistant cell line (Additional files 8 and 10). Again, just a few genes displaying copy number alterations were shared between the two resistant clones derived from ZR-75-1 and BT-474, implying genetic clonal divergence.

\section{T-47D tamoxifen-resistant cells develop shared as well as individual sensitivities}

To reveal the pharmacogenomic relationships between the drug response profiles and the genetic alterations, we integrated the drug response and genetic profiling data. Specifically, based on the DSS differences, we first selected the most pronounced drug response changes between each parental-resistant cell line pair. To correlate the genomic alterations with the drug responses we then constructed sensitivity and resistance networks among the target genes of the selected drugs, and mapped the point mutations and CNV changes onto these molecular networks as detailed in Materials and Methods, Construction of drug sensitivity and coresistance networks. We also merged the obtained networks with the IPA canonical pathway for Estrogen Receptor Signaling. However, only in two out of fourteen networks more than two overlapping molecules were found, indicating that only a fraction of them are actually connected with the ER signaling pathway and hence, in the majority of cases, the sensitivity and resistance mechanisms arise independently of ER. Overall, the number of genetic alterations mapping onto the drug target networks varied between 11 (T-47D Tam2 sensitivity network) and 0 (some ZR-75-1 and BT-474 networks), and converged into a number of target genes. In all but two networks (BT-474 Tam2 and ZR-75-1 Tam2 sensitivity) all the observed CNV changes were heterozygous deletions (Figs. 3, 4, 5 and 6 and Additional files 11 and 12). Of the two tamoxifen-resistant clones generated from the parental T-47D, both exhibited mostly sensitized profiles. T-47D Tam1 and Tam 2 both acquired sensitivity towards the SRC/ABL-family inhibitors; the Tam1 cells towards three of them: ponatinib, dasatinib and KX2-391; and the Tam2 cells against two of them: dasatinib and KX2-391 (Figs. 3 and 4). The Tam1 cells also became sensitive towards selumetinib, a MEK inhibitor, and the Tam2 cells towards VX-11E, an ERK1/2 inhibitor. In addition, sensitivity towards BCL-family inhibitors (navitoclax, obatoclax), and RAF inhibitors (regorafenib, RAF265) was observed in Tam1, but not in Tam2. Reflecting the acquired drug sensitivities, multiple target genes of these drugs or their upstream effectors converged into the same sensitivity networks, and notably, many of these target genes also harbored genetic changes.

\section{Increased sensitivity towards HER2/EGFR inhibitors}

Similar to the T-47D resistant clones, the tamoxifenresistant BT-474 s displayed mainly acquisition of drug sensitivities. However, unlike any of the other tamoxifen- 


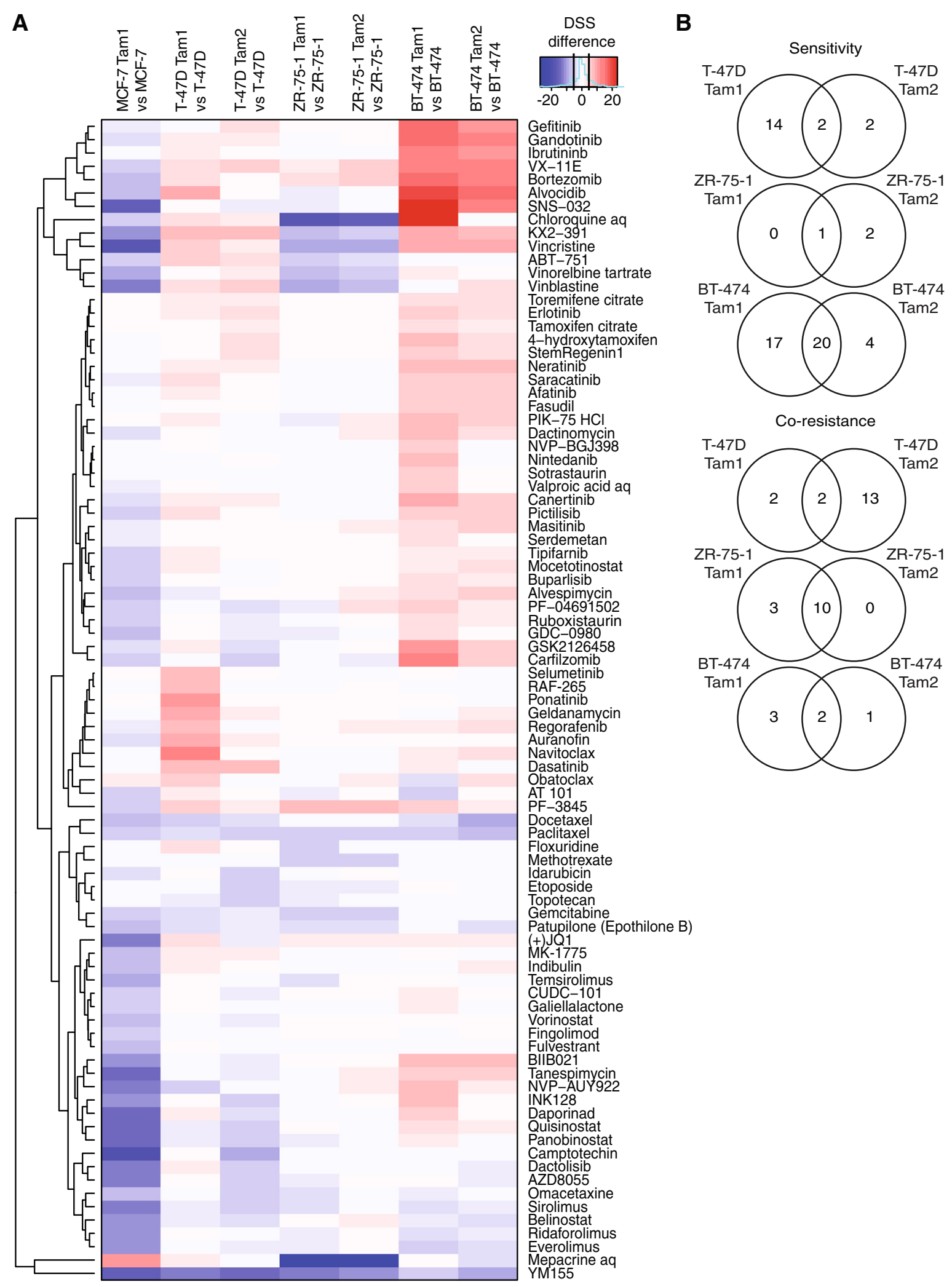

Fig. 2 (See legend on next page.) 
(See figure on previous page.)

Fig. 2 Tamoxifen-resistant cells display distinct drug response profiles. a Hierarchical clustering and heat map visualization of the drug sensitivity score (DSS) differences of each resistant/parental cell line pair. Red (positive DSS difference) represents sensitivity and blue (negative DSS difference) co-resistance. Drugs with DSS difference $<5$ in all of the comparisons were omitted. Black lines in the color bar highlight the cutoff $(5,-5)$. b Tamoxifen-resistant clones derived from same parental cells display distinct drug response profiles. Venn diagrams show overlap of drugs that the cells become sensitive or co-resistant towards. Drugs with DSS difference $<5$ were omitted

resistant clones, BT-474 Tam1 and Tam2 developed vulnerability to several EGFR/HER2-inhibitors (gefitinib, ibrutinib, neratinib, nintedanib; Fig. 5a, c and Fig. 6a, c). Notably, the parental BT-474 s also showed sensitivity towards HER2/EGFR inhibitors (Additional file 2), which was selectively enhanced upon developing resistance to tamoxifen. In addition, sensitivity towards cdk-inhibitors (SNS-032, alvocidib) emerged, and several of the drugs' target genes or their upstream effectors also harbored CNV changes (Fig. 6d). Both tamoxifen-resistant BT-474 clones also developed sensitivity towards the ERK1/2- inhibitor VX-11E and the JAK2-inhibitor gandotinib. BT-474 Tam2 also displayed gain of JAK2, possibly explaining the developing sensitivity towards gandotinib (Fig. 6d).

\section{Co-resistance against multiple drugs evolves across the tamoxifen-resistant cells}

In addition to emerging drug sensitivities, we also observed acquired co-resistances upon development of tamoxifen resistance. In contrast to the other resistant cells that developed both new sensitivities as well as co-

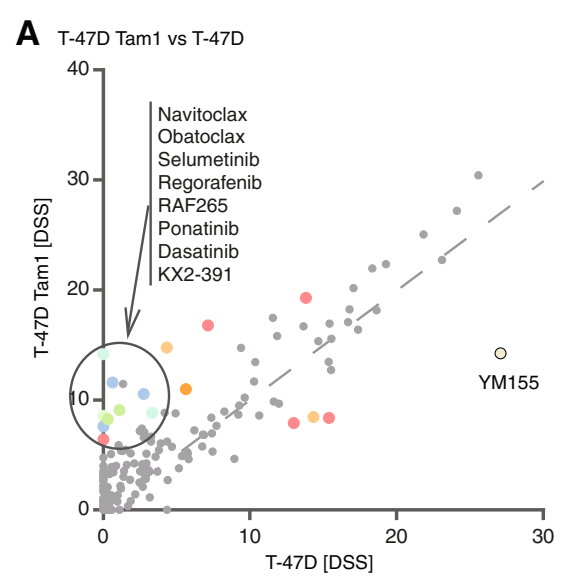

C

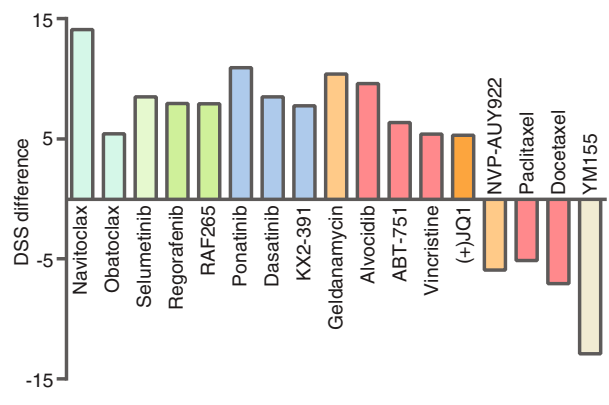

B

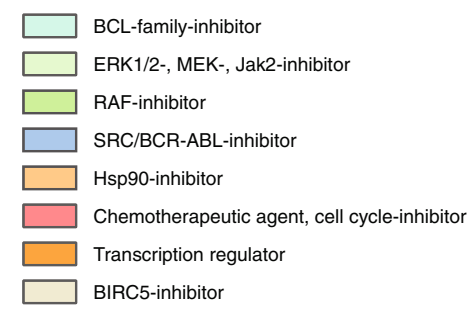

D

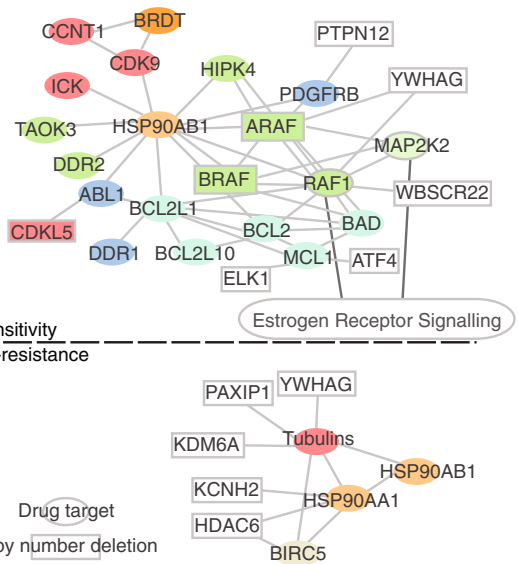

Fig. 3 Drug testing and molecular profiling reveal sensitivity and co-resistance networks in T-47D Tam1. a DSS differences of tamoxifen-resistant T-47D Tam1 vs parental cells reveal emerging sensitivities (above the dotted line) and co-resistances (below the dotted line) upon acquiring tamoxifen resistance. $\mathbf{b}$ Color legend of the drug target class. For visualization purposes, the drugs were colored according to their target class as indicated, and the coloring matched with their target genes. c Drugs with DSS difference $>5$. Positive values indicate sensitivity and negative co-resistance. $\mathbf{d}$ Matching of the drugs that the cells show acquired sensitivity or co-resistance towards with their specific target genes reveals molecular networks behind sensitivity and co-resistance in T-47D Tam1. Drugs without target genes in the networks are not displayed. Drug targets (colored) and upstream molecules (uncolored) are denoted as follows: ovals, molecules without genomic changes; rectangles with solid line, molecules with copy number deletions, high confidence ( $p<0,05$ and resistant/parental frequency $>30 \%)$ point mutations could not be connected to the network and are thus not displayed. Molecules that are connected with the ER signalling pathway are connected by a dark grey line to the boxed text "Estrogen Receptor Signalling" 


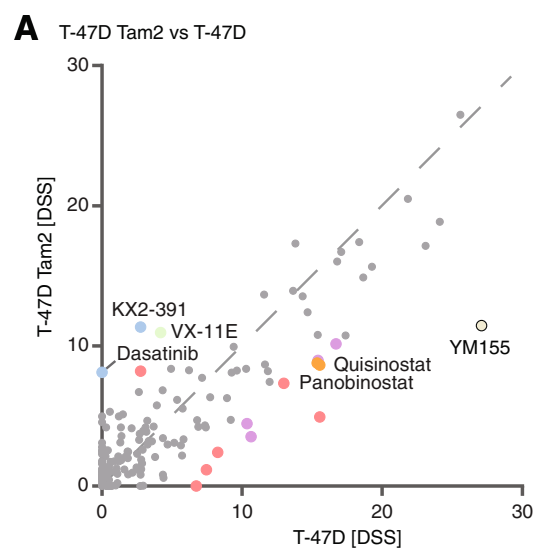

C

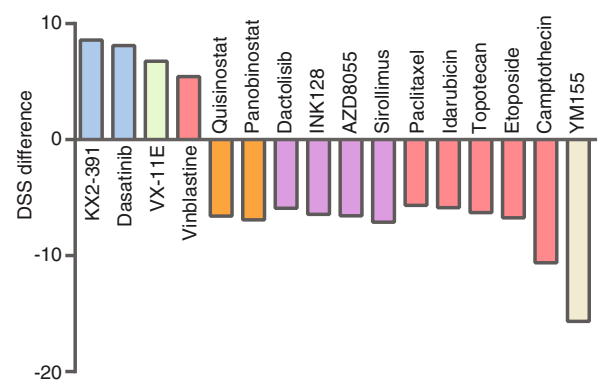

B

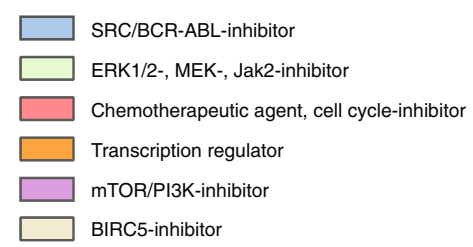

D
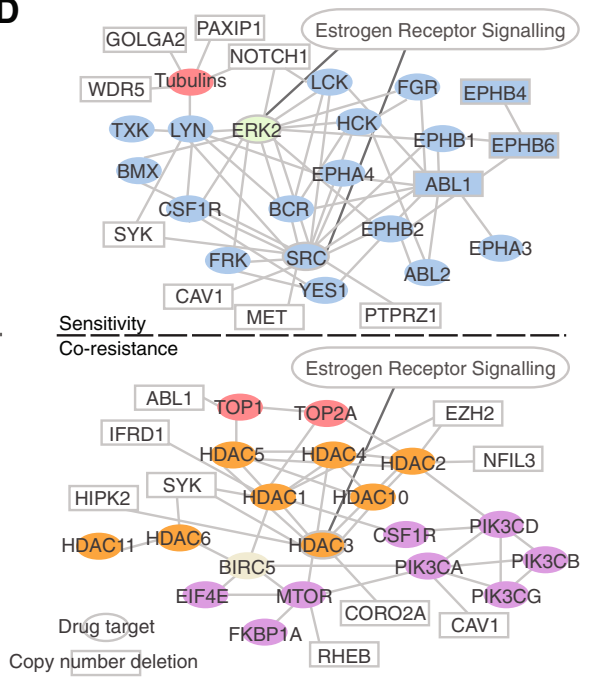

Fig. 4 Drug testing and molecular profiling reveal sensitivity and co-resistance networks in T-47D Tam2. a DSS differences of tamoxifen-resistant T-47D Tam2 vs parental cells reveal emerging sensitivities (above the dotted line) and co-resistances (below the dotted line) upon acquiring tamoxifen resistance. $\mathbf{b}$ Color legend of the drug target class. For visualization purposes, the drugs were colored according to their target class as indicated, and the coloring matched with their target genes. c Drugs with DSS difference $>5$. Positive values indicate sensitivity and negative co-resistance. $\mathbf{d}$ Matching of the drugs that the cells show acquired sensitivity or co-resistance towards with their specific target genes reveals molecular networks behind sensitivity and co-resistance in T-47D Tam2. Drugs without target genes in the networks are not displayed. Drug targets (colored) and upstream molecules (uncolored) are denoted as follows: ovals, molecules without genomic changes; rectangles with solid line, molecules with copy number deletions, high confidence $(p<0,05$ and resistant/parental frequency $>30 \%)$ point mutations could not be connected to the network and are thus not displayed. Molecules that are connected with the ER signalling pathway are connected by a dark grey line to the boxed text "Estrogen Receptor Signalling"

resistances, the tamoxifen-resistant MCF-7 Tam1 displayed an overwhelmingly co-resistant drug response profile, including resistance towards many chemotherapeutics (camptothecin, vincristine, SNS-032), but also several mTOR- and two HDAC-inhibitors (dactolisib, AZD8055, sirolimus, panobinostat, belinostat) (Additional file 11). In the two T47-D tamoxifen clones, the resistance networks were markedly different, with T47-D Tam1 displaying resistance merely to four agents, two of which were chemotherapeutics, whereas T-47D Tam2 cells exhibited co-resistance to a large variety of drugs. These included some of the same drugs as for the Tam 1 clone, and additionally Tam 2 showed resistance to four mTOR- and two HDAC-inhibitors (Figs. 3 and 4). Both ZR-75-1 as well as the BT-474 resistant clones developed co-resistance to several chemotherapeutics (Figs. 5 and 6, Additional files 5, 6, 12), with the ZR-75-1 networks being nearly identical, reflecting the high similarity between their drug response patterns. Interestingly, the BT-474 Tam1 cells additionally showed resistance to rapamycin and everolimus, two mTOR inhibitors, as well as to AT 101, a BCL-family inhibitor (Fig. 5). Collectively, compared to the sensitivity profiles, the resistance networks demonstrated less variance between the different tamoxifen-resistant clones, with the majority of them developing co-resistance towards common chemotherapeutics (Table 1).

\section{Shared sensitivities and co-resistances}

We next addressed the development of common sensitivities and co-resistances upon acquisition of tamoxifen resistance. Cross-comparison of the drug response profiles across all tamoxifen-resistant clones revealed that several of them developed sensitivity towards the ERK1/ 

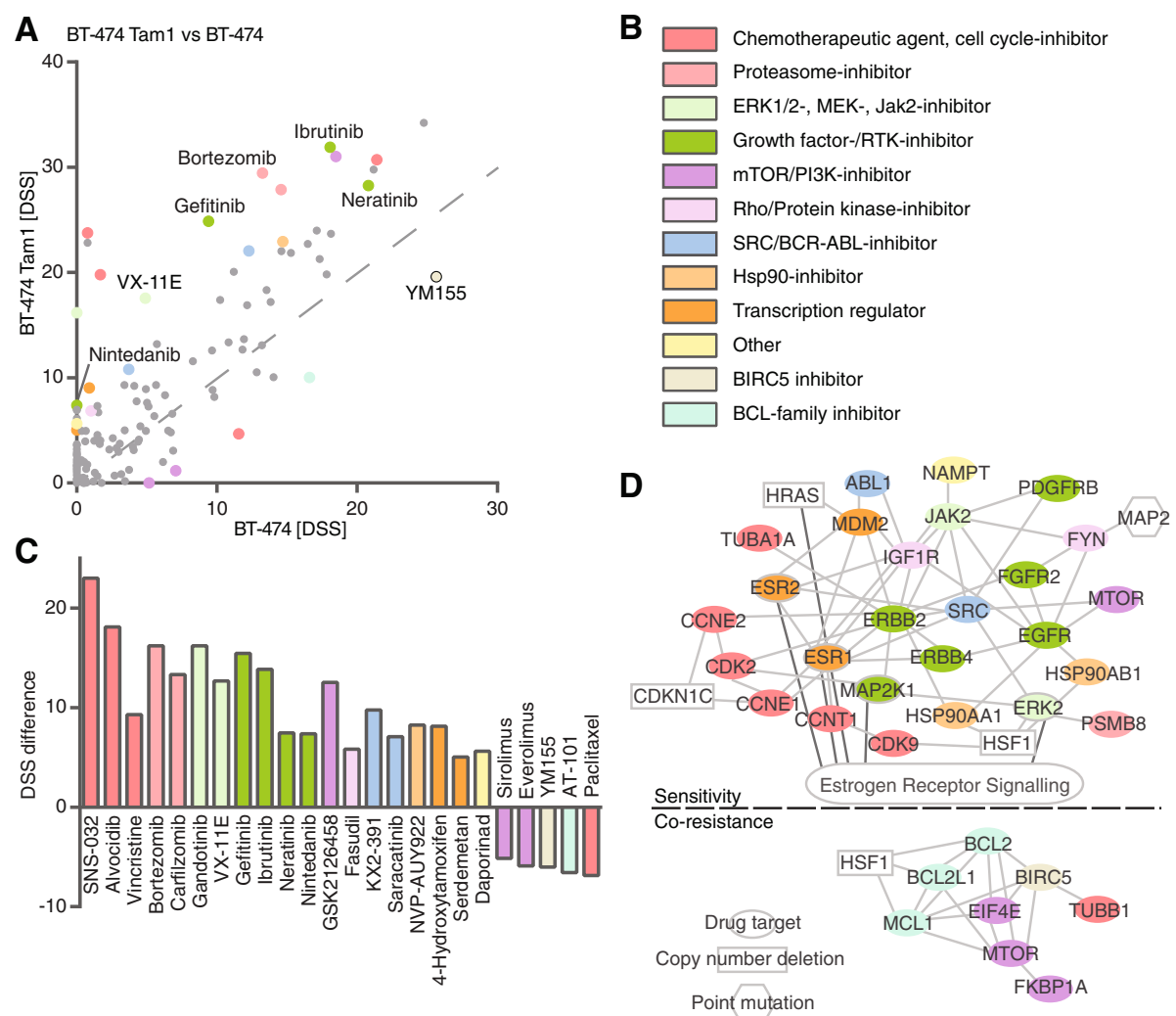

Fig. 5 Drug testing and molecular profiling reveal sensitivity and co-resistance networks in BT-474 Tam1. a DSS differences of tamoxifen-resistant BT-474 Tam1 vs parental cells reveal emerging sensitivities (above the dotted line) and co-resistances (below the dotted line) upon acquiring tamoxifen resistance. $\mathbf{b}$ Color legend of the drug target class. For visualization purposes, the drugs were colored according to their target class as indicated, and the coloring matched with their target genes. c Drugs with DSS difference $>5$. Positive values indicate sensitivity and negative co-resistance. $\mathbf{d}$ Matching of the drugs that the cells show acquired sensitivity or co-resistance towards with their specific target genes reveals molecular networks behind sensitivity and co-resistance in BT-474 Tam1. Drugs without target genes in the networks are not displayed. Drug targets (colored) and upstream molecules (uncolored) are denoted as follows: ovals, molecules without genomic changes; rectangles with solid line, molecules with copy number deletion, and polygons molecules with high confidence $(p<0,05$ and resistant/parental frequency $>30 \%)$ point mutations. Molecules that are connected with the ER signalling pathway are connected by a dark grey line to the boxed text "Estrogen Receptor Signalling"

2-inhibitor VX-11E, the proteasome-inhibitor bortezomib, and the FAAH-inhibitor PF-3845. Common coresistance towards the survivin-inhibitor YM155 and the chemotherapeutic agent paclitaxel also occurred (Fig. 7a). Furthermore, even with the limited sample size, these shared responses were statistically significant (rank product analysis, Fig. 7b). To further assess the EGFR/ERK signaling pathway in the resistant cells, we selected the MEK/ERK inhibitors for which a differential drug sensitivity score was obtained, i.e. VX-11E and selumetinib. The concentration range was selected based on the $\mathrm{IC}_{50}$ of the drugs in the individual cell lines (Additional file $5)$. We then cultured the cells either in their default culture media, or with increasing concentrations of VX$11 \mathrm{E}$, or with VX-11E and selumetinib concomitantly, and performed Western blotting with ERK1/2 and EGFR antibodies (Fig. 7c). The basal levels of these unphosphorylated as well as phosphorylated signaling proteins were lowered in the tamoxifen-resistant cells compared to the parentals (Fig. 7c), but upon increasing concentrations of VX-11E, slight increase in phosphorylated ERK1/2 was observed in the T-47Ds. However, upon additional inhibition of MEK with selumetinib, the increase in phosphorylated ERK1/2 was diminished.

\section{Discussion}

In the present study, we report the development and systematic characterization of seven long-term tamoxifentreated cell lines, and by pharmacogenomic profiling, determine the drug response profiles and mutational landscapes of these drug-resistant models. Different in vitro and in vivo models of endocrine-resistance have been developed to explore common mechanisms behind resistance development [9-11, 19, 20, 22, 40-50] However, to our knowledge, this is the first comprehensive drug testing study with hundreds of oncology compounds across a 

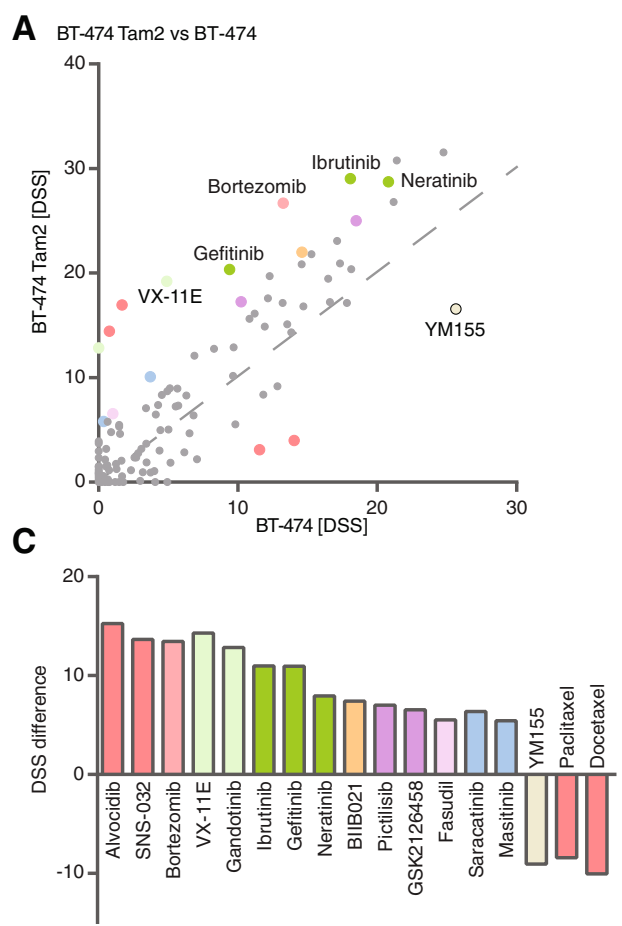

B

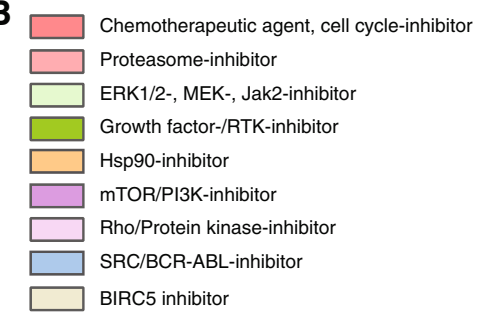

D

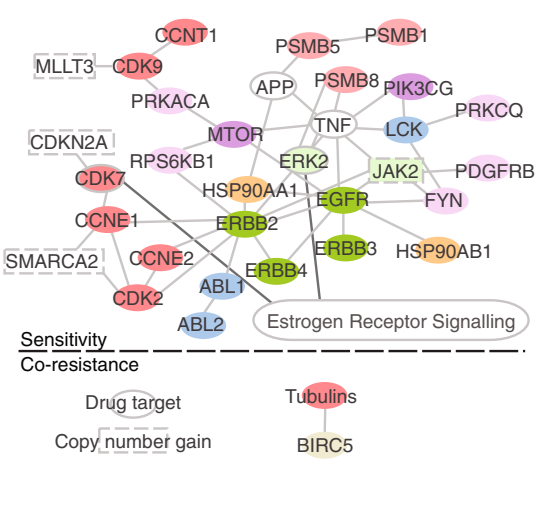

Fig. 6 Drug testing and molecular profiling reveal sensitivity and co-resistance networks in BT-474 Tam2. a DSS differences of tamoxifen-resistant BT-474 Tam2 vs parental cells reveal emerging sensitivities (above the dotted line) and co-resistances (below the dotted line) upon acquiring tamoxifen resistance. $\mathbf{b}$ Color legend of the drug target class. For visualization purposes, the drugs were colored according to their target class as indicated, and the coloring matched with their target genes. c Drugs with DSS difference $>5$. Positive values indicate sensitivity and negative co-resistance. $\mathbf{d}$ Matching of the drugs that the cells show acquired sensitivity or co-resistance towards with their specific target genes reveals molecular networks behind sensitivity and co-resistance in BT-474 Tam2. Drugs without target genes in the networks are not displayed. Drug targets (colored) and upstream molecules (uncolored) are denoted as follows: ovals, molecules without genomic changes; rectangles with dashed line, molecules with copy number gain, high confidence $(p<0,05$ and resistant/parental frequency $>30 \%)$ point mutations could not be connected to the network. Molecules that are connected with the ER signaling pathway are connected by a dark grey line to the boxed text "Estrogen Receptor Signalling"

panel of several tamoxifen-resistant models. Using this approach, we identify clone-specific molecular networks reflecting the diversity of pathways leading to endocrine resistance. It is noteworthy that as the availability of clinical data sets on diagnosed acquired tamoxifen resistance with response/survival data are essentially non-existent to date, the resistant/sensitive cell line models and associated data sets presented here form a valuable research resource.

Concurrently with developing tamoxifen resistance, novel drug vulnerabilities emerge. Here, we identified common, cell type-, and cell clone-specific sensitivities. The most important of these are listed in Table 1. Additionally, several of the sensitizing drugs are in clinical trials for treatment of advanced or metastatic breast cancer. However, possible correlation between patient enrollment criteria, observed molecular mechanisms and the sensitivities and co-resistances identified here remains to be investigated.

All resistant cell lines except one (MCF-7 Tam1) exhibited gained sensitivity towards the ERK1/2 inhibitor
VX-11E. ERK1/2 inhibition prevents its autophosphorylation $[10,51]$ and results in reduced phosphorylation and thereby also decreased activation of ER [9]. Overactivity of ERK1/2 has been shown to associate with loss of ER, and decreased levels of ER are also seen in the majority of our tamoxifen-resistant cell lines (Additional file 4) [52]. However, the basal levels of unphosphorylated or phosphorylated EGFR/ERK are not elevated in the tamoxifen resistant lines; rather the opposite, i.e. decrease in basal levels as well as dephosphorylation of ERK1/2 and EGFR is observed (Fig. 7c and data not shown). We therefore anticipate that increased phosphorylation of these signaling proteins does not explain the observed sensitivity towards VX-11E. Interestingly, upon increasing concentrations of VX-11E, slight increase in phosphorylated ERK1/2 is observed in the T47D Tam clones. However, upon additional inhibition of MEK with selumetinib, this effect is diminished. The effect of VX-11E inducing ERK1/2 phosphorylation has also previously been reported [53] and might therefore reflect a general mode of action especially as the same 
Table 1 Tamoxifen-resistant cells develop individual as well as common drug sensitivities and co-resistances. Sensitizing and desensitizing drugs, drug target classes, specific target genes and as well as affected cell lines are listed

\begin{tabular}{|c|c|c|c|}
\hline Sensitivity towards & Drug target class & $\begin{array}{l}\text { Specific } \\
\text { target gene }\end{array}$ & $\begin{array}{l}\text { Observed in } \\
\text { cell line }\end{array}$ \\
\hline \multirow[t]{2}{*}{ Navitoclax, Obatoclax } & \multirow[t]{2}{*}{ BCL-family } & BCL2L1 & \multirow[t]{2}{*}{ T-47D Tam1 } \\
\hline & & $B A D$ & \\
\hline RAF265, Ponatinib & RAF-family & RAF1 & T-47D Tam1 \\
\hline \multirow[t]{2}{*}{ Dasatinib, KX2-391 } & \multirow[t]{2}{*}{$\mathrm{SRC} / \mathrm{ABL}$} & ABL1 & \multirow{2}{*}{$\begin{array}{l}\text { T-47D Tam1 } \\
\text { \& Tam2 }\end{array}$} \\
\hline & & SRC & \\
\hline$V X-11 E$ & MAPK1 & MAPK1 & $\begin{array}{l}\text { T-47D Tam2, } \\
\text { BT-474 Tam2, } \\
\text { ZR-75-1 Tam2 }\end{array}$ \\
\hline \multirow{3}{*}{$\begin{array}{l}\text { Gefitinib, Ibrutinib, } \\
\text { Neratinib, Nintedanib }\end{array}$} & \multirow[t]{3}{*}{ HER2/EGFR } & ERBB2 & \multirow{3}{*}{$\begin{array}{l}\text { BT-474 Tam1 } \\
\& \text { Tam2 }\end{array}$} \\
\hline & & ERBB4 & \\
\hline & & ERBB3 & \\
\hline \multirow[t]{4}{*}{ Vinblastine } & \multirow[t]{4}{*}{ Tubulins } & TUBA1A & \multirow[t]{4}{*}{ T-47D Tam2 } \\
\hline & & TUBA4A & \\
\hline & & TUBA1C & \\
\hline & & TUBB6 & \\
\hline PF-3845 & $\mathrm{FAAH}$ & FAAH & ZR-75-1 Tam2 \\
\hline Bortezomib & PSMB-family & PSMB5 & ZR-75-1 Tam2 \\
\hline Resistance towards & Drug target class & $\begin{array}{l}\text { Specific } \\
\text { target gene }\end{array}$ & $\begin{array}{l}\text { Observed in } \\
\text { cell line }\end{array}$ \\
\hline YM155 & $\mathrm{BIRC5}$ & $\mathrm{BIRC5}$ & $\begin{array}{l}\text { T-47D Tam1 } \\
\text { \& Tam2, } \\
\text { MCF-7 Tam1, } \\
\text { BT-474 \& } \\
\text { Tam2 }\end{array}$ \\
\hline \multirow{2}{*}{$\begin{array}{l}\text { Quisinostat, } \\
\text { Panobinostat }\end{array}$} & \multirow[t]{2}{*}{ HDACs } & $\mathrm{HDAC1}$ & \multirow{2}{*}{$\begin{array}{l}\text { T-47D Tam2, } \\
\text { MCF-7 Tam1 }\end{array}$} \\
\hline & & HDAC6 & \\
\hline \multirow{6}{*}{$\begin{array}{l}\text { Docetaxel, } \\
\text { Paclitaxel }\end{array}$} & \multirow[t]{6}{*}{ Tubulins } & TUBA1C & \multirow[t]{6}{*}{ BT-474 Tam2 } \\
\hline & & TUBB2A & \\
\hline & & TUBB3 & \\
\hline & & TUBB & \\
\hline & & TUBB4B & \\
\hline & & TUBB6 & \\
\hline $\begin{array}{l}\text { Temsirolimus, } \\
\text { Everolimus, } \\
\text { Ridaforolimus, } \\
\text { INK128, Sirolimus, } \\
\text { AZD8055, Dactolisib }\end{array}$ & mTOR & mTOR & MCF-7 Tam1 \\
\hline
\end{tabular}

effect is observed also in our parental cells (Fig. 7c). It could therefore be speculated that already a short-term tamoxifen treatment causes an effect on the levels of phosphorylated ERK1/2 and that long-term exposure leads to, at least partial, down-regulation of EGFR and ERK1/2. As cells are challenged with increasing concentrations of an ERK1/2 inhibitor (VX-11E), an increase in ERK1/2 phosphorylation is seen, with concomitant cell killing of the tamoxifen-resistant cells observed in the drug screen. However, further studies to elucidate the exact mechanisms are needed.

We also identified bortezomib, a proteasome inhibitor, as a sensitizing agent (Figs. 5, 6 and 7 and Additional file 12, Table 1). A direct role for bortezomib in reversing tamoxifen resistance has not been demonstrated before, although a link between proteasome function and estrogen receptor -mediated transcription has been suggested [54], and bortezomib has recently been shown to enhance endocrine treatment in cell line models as well as in humans [11-13].

In addition to shared sensitivity to VX-11E and bortezomib in the tamoxifen-resistant cells, we also identified cell line specific drug sensitivities (Table 1, Figs. 3, 4, 5 and 6, Additional files 11 and 12). T-47D Tam1 and Tam2 cells displayed sensitivity towards the SRC-family inhibitor KX2-391 and the dual ABL/SRC-inhibitors dasatinib and ponatinib (Figs. 3 and 4). This is in agreement with recent findings $[20,55]$. Another SRCinhibitor, SU6656, has also been reported to inhibit growth of tamoxifen-resistant cells [42], highlighting the potential of SRC-inhibition in overcoming endocrine resistance. Interestingly, dasatinib has been shown to overcome tamoxifen resistance in MCF-7/fibroblast coculture, and it is currently undergoing clinical trials on metastasized ER-positive breast cancer.

Upon acquiring resistance to tamoxifen, the T-47D Tam 1 cells also gained sensitivity towards the BCLfamily inhibitors navitoclax and obatoclax (Fig. 3). BCL2 family proteins BCL-2, BCL2L1, BCL2L10 and MCL1, represented in the network, are major negative regulators of apoptosis, and thus, upregulation of their expression might offer the tamoxifen-challenged cells means to overcome resistance, as well as downregulation of BAD, a proapoptotic regulator. Indeed, $\mathrm{BCL}-2$ has been indicated in tamoxifen resistance, and consequently, a BCL2 inhibitor, ABT-737, has been reported to restore sensitivity [56]. Additionally, tamoxifen treated patients with low level of BAD expression had a worse prognosis [57]. The T-47D Tam1 cells also displayed sensitivity towards RAF-inhibitors BAY 73-4506 and RAF265. This is in line with previous findings on overexpression of $R A F 1$ promoting tamoxifen-resistant growth [58]. Both navitoclax and BAY 73-4506 are being investigated for treatment of different cancers, navitoclax for lung cancer and lymphoma, and BAY 73-4506 for metastatic colorectal cancer among others. Our results, and those from others [56-58] suggest that BCL- and RAF-inhibitors might offer means to treat also endocrine-resistant breast cancer.

We also identified sensitizers with preference for the luminal B-derived tamoxifen-resistant cells, BT-474 Tam 1 and Tam2. These included the cdk-inhibitors 
A

Sensitivity

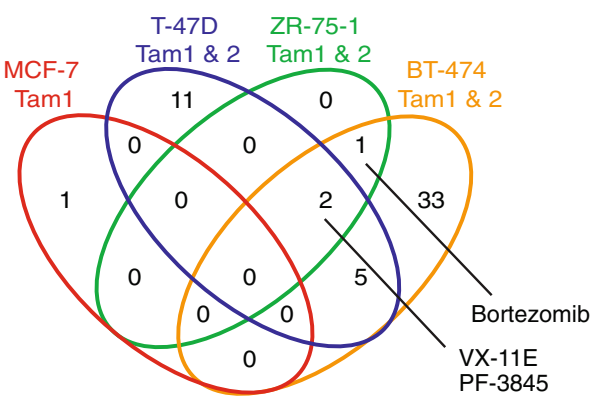

B

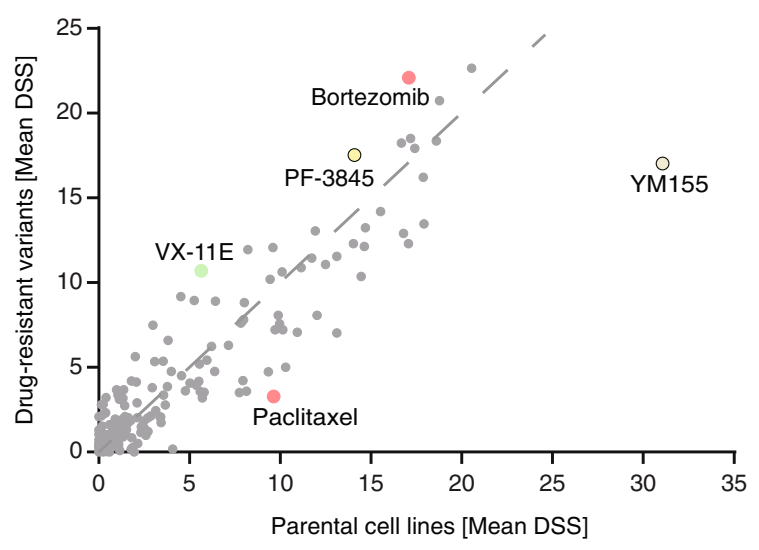

Co-resistance

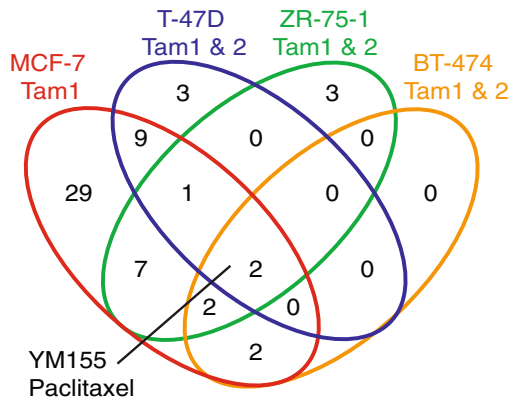

C

kDa

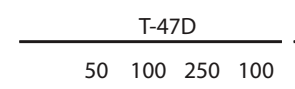

$-$

T-47D Tam

(1)

$175-\square--\frac{1}{-}$

$175-$

$66-00$

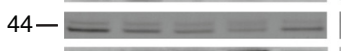

$44-\square=$

$\begin{array}{llll}50 & 100 & 250 & 100\end{array}$

$\frac{\text { T-47D Tam2 }}{50 \quad 100250 \quad 100}$
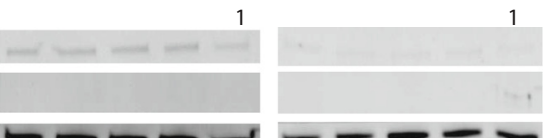

X (nM)

Selumenitib $(\mu \mathrm{M})$

EGFR

phospho EGFR

$-0-0$

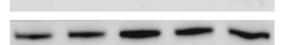

ER alpha

ERK1/2

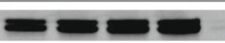

$==$

phospho ERK1/2

pactin

\begin{tabular}{lll} 
BT-474 & \\
\hline $50 \quad 100 \quad 250 \quad 100$
\end{tabular}

BT-474 Tam1

BT-474 Tam2

$\begin{array}{lllll}100 & 50 & 100 & 250 & 100\end{array}$

$\begin{array}{llll}50 & 100 \quad 250 \quad 100\end{array}$

VX-11E (nM)

1 Selumenitib $(\mu \mathrm{M})$

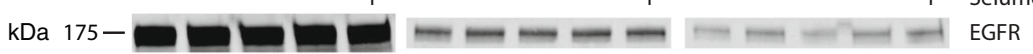

175- -

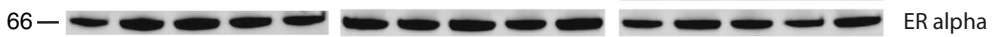

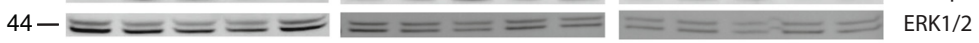

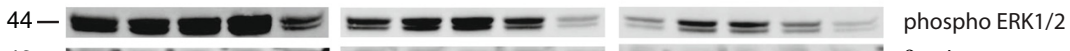

$42-100-3$

Fig. 7 Tamoxifen-resistant cells develop common drug sensitivities and co-resistances. a Venn diagrams illustrate shared drug sensitivities and co-resistances of drugs with a DSS difference of at least 5. b Rank-product analysis of Drug Sensitivity Scores (DSS) between tamoxifen-resistant and parental cell lines identified statistically significant shared sensitizing and desensitizing drugs. c Western blotting of EGFR, pEGFR, ERK1/2, pERK1/2, ERa and Bactin under increasing concentrations of VX-11E and $1 \mu \mathrm{M}$ selumetinib of T-47D and BT-474 isogenic cell lines. Resistant cell lines were cultured in media supplemented with $1 \mu \mathrm{M}$ tamoxifen

SNS-032 and alvocidib, along with the EGFR-inhibitor gefinitib and the Btk-inhibitor PCI-32765, and several HER2/EGFR-inhibitors (Figs. 5 and 6). Crosstalk between ER and ERBB2/EGFR pathways has been shown to be activated in tamoxifen resistance [59]. Recently, the EGFR/HER2 dual inhibitor AZD8931 was also 
suggested to inhibit growth of MCF-7 or T-47D tamoxifen-resistant cells in xenograft models [60]. It is noteworthy that in our study, the parental BT-474 cells, unlike all others presented here, initially amplify and overexpress HER2, and display some sensitivity towards HER2/EGFR-inhibitors (Additional file 2). Interestingly, as tamoxifen resistance develops, the cells become more sensitive to several of the HER2/EGFR-inhibitors and indeed, the combined use of growth factor receptor kinase inhibitors in conjunction with tamoxifen has been suggested to circumvent endocrine resistance [45], and combination therapy with antihormone and gefinitib has demonstrated resensitization to tamoxifen in xenografts $[61,62]$. However, our results on decreasing EGFR / phospho-EGFR levels upon acquired resistance (Fig. 7c) indicate that mechanisms other than direct upregulation of the EGFR pathway are responsible for the observed gained sensitivity.

Development of primary drug resistance in cancer treatment frequently results in the emergence of secondary resistances. Here, we discovered that upon acquiring tamoxifen resistance, all of the resistant cells acquired co-resistance towards at least one chemotherapeutic agent, such as paclitaxel, docetaxel, vincristine, vinblastine or topotecan (Figs. 2, 3, 4, 5, 6 and 7, Table 1 and Additional files 5, 11 and 12). Even though chemoresistance has been associated with the estrogen receptor [24], the co-resistance observed here may rather reflect the slowed-down growth of many of the resistance clones, and may therefore propose a uniform mechanism for paclitaxel resistance (Additional file 3). However, selective co-resistance still occurs, as the cells do not become universally co-resistant against all chemotherapeutics. Nevertheless, general down-regulation of cellular functions is especially evident with the tamoxifenresistant MCF-7 Tam1 cells, which not only possess an overwhelmingly co-resistant drug response profile, but also down-regulate cell signaling (Additional files 3, 4, 11). Indeed, already a short-term tamoxifen-treatment of MCF-7 cells triggers a predominant down-regulation of gene expression [63], suggesting that depending on the molecular background, some tamoxifen-resistant cells might exhibit an intrinsically more unresponsive profile.

Interestingly, every single tamoxifen-resistant cell line was also more resistant to the survivin (BIRC5-) inhibitor YM155 than their parental counterparts (Figs. 2, 3, 4, 5, 6 and 7, Table 1 and Additional files 5, 11 and 12), suggesting a role for survivin in development of tamoxifen resistance. Survivin has recently been associated with resistance to chemo- or hormonal therapy, and has been identified to predict poor clinical outcome via ERBB2mediated overexpression [47]. Furthermore, siRNAknock down of BIRC5 has been shown to enhance cell sensitivity to tamoxifen [64]. Alternatively, it has been speculated that uptake of YM155 is dependent on cell membrane a solute carriers, encoded by the SLC35F2 gene [65]. Upon resistance development, expression of the solute carriers possibly decreases and consequently, less YM155 enters the cells making them resistant to the drug.

As initiation and development of acquired tamoxifen resistance are largely thought to be driven by genetic adaptations $[3-5,7]$ we inspected the genetic landscape of the drug-resistant cells by exome-sequencing and correlated the findings with our drug profiling data. Tamoxifen-resistant cells accumulated point mutations and copy number changes throughout their genomes, with only some of the changes being common between two resistant clones originating from the same parental cells, implying clonal divergence (Additional files 8, 9 and 10). Whilst many of the genetic aberrations that have been associated with endocrine resistance previously were also recapitulated here, our data as a whole demonstrate that new sensitivities may develop largely independent of the genetic changes, and in fact, antiestrogen resistance can be seen even in the absence of any evident mutations [66]. Analogous phenomenon has been noted in leukemic cells [67]. Accumulation of numerous genomic aberrations can trigger resistance development [20], but mutations can also be carried along as passengers as a result of selection pressure, rather than emerge as true evolutionary drivers [68]. The data presented here demonstrate that in the majority of cases, no single genetic alteration can be identified as responsible for the drug response, but on the contrary, multiple target genes of the drugs converge into the same response networks, and many of these target genes also harbor genetic changes. Therefore, resistance development is likely to involve complex interactions comprising genetic as well as transcriptional and epigenetic mechanisms, or other adaptive changes in cell signaling.

\section{Conclusion}

Taken together, the results presented in this study demonstrate that upon acquiring endocrine-resistance, breast cancer cells follow different paths to resistance, as shown by distinct genomic evolution. As a consequence, gained sensitivity as well as co-resistance towards a variety of other agents evolves. In addition to common vulnerability towards ERK1/2- and proteasome-inhibitors, we also identified a universal co-resistance towards the survivininhibitor YM155 in tamoxifen-resistant cells. Drug response profiles between cell clones derived from the same parental cells differed markedly, and different members of the same drug classes could be either sensitizing or desensitizing. This suggests that resistance mechanisms vary within tumors, among patients and with time, highlighting the need for personalized diagnosis and clone-targeting 
therapies in the treatment of tamoxifen-resistant breast cancer. As shown here, prediction of drug responses can be difficult based on genomic profiling alone. This study provides a reference set of materials (drug-resistant cell lines), and their cell biological, genomic and drug response profiling for future studies aiming to test novel therapies for breast cancer with acquired tamoxifen resistance.

\section{Additional files}

Additional file 1: Primer sequences. (XLSX $10 \mathrm{~kb})$

Additional file 2: Parental cells show sensitivity and selectivity towards known breast cancer drugs according to their subtype. (A) Drug Sensitivity Scores (DSS) of known breast cancer treatment drugs. (B) DSS of drugs specific to luminal A-(left) and luminal B-subtypes (right). Results are extracted from the data on all drugs tested in all cell lines, presented in Additional file 5. The drugs specific to parental luminal $A$ and $B$ subtypes were identified based on their DSS scores. (PDF $423 \mathrm{~kb}$ )

Additional file 3: Growth and tamoxifen-tolerance of tamoxifen-resistant cells. (A) CTG-viability measurement of tamoxifen-resistant vs parental cells treated with increasing concentrations of tamoxifen. (B) Measurement of active DNA synthesis by FACS-analysis showing accumulation of resistant cells in the G0/G1 phase of the cell cycle. (PDF $1035 \mathrm{~kb}$ )

Additional file 4: Estrogen responsivity of ERa-mediated transcription in the tamoxifen-resistant cells. (A) Quantitative RT-PCR showing decreased ERa target gene expression in the resistant cells. (B) Quantitative RT-PCR of the ERa target gene pS2 expression upon estradiol withdrawal and subsequent addition of estradiol back to the cells. (C) Western blotting displaying altered protein levels of ERa in the resistant cells. A.u. = arbitrary units, E2 = 17ß-estradiol. Error bars show standard deviation. (PDF 704 kb)

Additional file 5: Drug Sensitivity Scores. Drugs used in the study, their approval status and Drug Sensitivity Scores (DSS) of the tamoxifen-resistant and their parental cell lines. Max.Conc $[\mathrm{nM}]=$ maximum concentration, Min.Conc $[\mathrm{nM}]=$ minimum concentration, $\mathrm{MAX}=$ maximum \% inhibition, D1 [\% inhibition]... D5 [\% inhibition] = \% inhibition from the lowest (D1) to the highest drug concentration (D5). (XLSX $478 \mathrm{~kb}$ )

Additional file 6: DSRT statistics. (XLSX $9 \mathrm{~kb}$ )

Additional file 7: Exome-sequencing reads. (XLSX $10 \mathrm{~kb}$ )

Additional file 8: Copy number alterations and point mutations are scattered throughout the genomes of the tamoxifen-resistant cells. (A) Relative copy number of each resistant cell line measured by exome-sequencing and plotted as log2 ratio of resistant vs parental cell line (colored dots). Copy number gains/amplifications and losses/deletions are visible as peaks and valleys in the red segmentation line, respectively. Chromosomes are numbered and highlighted in alternating colors. High confidence point mutations ( $p<0,05$ and resistant/parental frequency $>30 \%$ ) are depicted above the segmentation line. (B) Venn diagrams show overlap of point mutations (black) and genes altered by CNVs (red) between the tamoxifen-resistant clones derived from same parental cells. (PDF $735 \mathrm{~kb}$ )

Additional file 9: Point mutations from exome-sequencing. Point mutations, sequencing reads and mutation frequency in tamoxifenresistant cells (Resistant Reference Reads, Resistant Variant Reads, Resistant Variant Frequency) compared to their parental cell lines (Parental Reference Reads, Parental Variant Reads, Parental Variant Frequency) revealed by exome-sequencing. Sample ID, chromosome, position, reference and variant base, affected gene, effect, effect impact, and $p$-values are also indicated. (XLSX $250 \mathrm{~kb}$ )

Additional file 10: Copy number variations from exome-sequencing Copy number changes in tamoxifen-resistant cells compared to their parental cell lines as revealed by exome-sequencing. Ensembl gene IDs, Hgnc symbols, chromosomal position (Chr, start, end), copy number value (copy_num), copy number status (cn_status) and potential presence at a breakpoint are indicated. (XLSX $8980 \mathrm{~kb})$
Additional file 11: Drug testing and molecular profiling reveal sensitivity and co-resistance networks in MCF-7 Tam1. (A) DSS differences of tamoxifenresistant MCF-7Tam1 vs parental cells reveal emerging sensitivities (above the dotted line) and co-resistances (below the dotted line) upon acquiring tamoxifen resistance. (B) Color legend of the drug target class. For visualization purposes, the drugs were colored according to their target class as indicated, and the coloring matched with their target genes. (C) Drugs with DSS difference $>5$. Positive values indicate sensitivity and negative co-resistance. (D) Matching of the drugs that the cells show acquired sensitivity or co-resistance towards with their specific target genes reveals molecular networks behind sensitivity and co-resistance in MCF-7 Tam1. Drugs without target genes in the networks are not displayed. Drug targets (colored) and upstream molecules (uncolored) are denoted as follows: ovals, molecules without genomic changes; rectangles with solid line, molecules with copy number deletions, high confidence $(p<0,05$ and resistant/parental frequency $>30 \%)$ point mutations could not be connected to the network and are thus not displayed. Molecules that are connected with the ER signalling pathway are connected by a dark grey line to the boxed text "Estrogen Receptor Signalling". (PDF $443 \mathrm{~kb}$ )

Additional file 12: Drug testing and molecular profiling reveal sensitivity and co-resistance networks in ZR-75-1 Tam1 and Tam2. (left) DSS differences of tamoxifen-resistant (A) ZR-75-1 Tam1 and (B) ZR-75-1 Tam2 vs parental cells reveal emerging sensitivities (above the dotted line) and co-resistances (below the dotted line) upon acquiring tamoxifen resistance. (middle) Drugs with DSS difference $>5$. Positive values indicate sensitivity and negative co-resistance. (right) Matching of the drugs that the cells show acquired sensitivity or coresistance towards with their specific target genes reveals molecular networks behind sensitivity and co-resistance in resistant cells. Drugs without target genes in the networks are not displayed. Drug targets (colored) and upstream molecules (uncolored) are denoted as follows: ovals, molecules without genomic changes; rectangles with dashed line, molecules with copy number gain, high confidence $(p<0,05$ and resistant/parental frequency $>30 \%$ ) point mutations could not be connected to the network and are thus not displayed. (B) Color legend of the drug target class. For visualization purposes, the drugs were colored according to their target class as indicated and the coloring matched with their target genes. Molecules that are connected with the ER signalling pathway are connected by a dark grey line to the boxed text "Estrogen Receptor Signalling". (PDF 464 kb)

\section{Abbreviations}

ER, estrogen receptor; DSRT, drug sensitivity and resistance testing; DSS, drug sensitivity score; KIBA-score, kinase inhibitor bioactivity -score; Ki, inhibitory constant; $\mathrm{Kd}$, dissociation constant; IC50, half maximal inhibitory concentration; IPA, ingenuity pathway analysis; CNV, copy number variation; cdk, cyclindependent kinases

\section{Acknowledgements}

The authors would like to express their sincerest thanks to Bhagwan Yadav, Swapnil Potdar, Dmitrii Bychkov, Ida Lindenschmidt and the whole FIMM Technology Centre personnel for their invaluable help in the drug testing and sequencing. Mariliina Arjama is acknowledged for excellent technical assistance, and Jing Tang for providing curated drug target (KIBA) data. This work was supported by Academy of Finland Post-Doctoral Researcher Grant (SK) and Research Grants 269862, 272437 and 279163 (TA) (www.aka.fi), the European Union's Seventh Framework Programme (FP7/2007-2013) under grant agreement n²58068; EU-FP7-Systems Microscopy NoE (SH), Academy of Finland Centre of Excellence, Integrative Life Science (ILS) doctoral program (AJ) (www.finbionet.fi/ils/), the Sigrid Juselius Foundation (http://sigridjuselius.fi), and the Cancer Society of Finland (www.cancer.fi).

\section{Availability of data and materials}

The datasets supporting the conclusions of this article are included within the article and its additional files. Raw exome-sequencing data have been deposited in the NCBI Sequence Read Archive [SRP: SRP050366].

\section{Authors' contributions}

SK designed the study and contributed to the generation and characterization of the cell lines, data analysis and writing of the manuscript. SH contributed to the characterization of the cell lines, drug sensitivity and resistance testing, data 
analysis and writing of the manuscript. AJ contributed to data integration and network analysis, HE contributed to CNV analysis, JPM provided the bioinformatical platform to run the data analysis, SE conducted the point mutation analysis. OB contributed to the generation of the cell lines. TA supervised the data integration and network analysis, and participated in manuscript writing and editing. OK supervised the entire project and participated in manuscript writing and editing. All authors read and approved the final manuscript.

\section{Competing interests}

The authors declare that they have no competing interests.

\section{Consent for publication}

Not applicable.

\section{Ethics approval and consent to participate}

Not applicable.

\section{Author details}

1 Institute for Molecular Medicine Finland (FIMM), Biomedicum 2U, Tukholmankatu 8, 00290 Helsinki, Finland. ${ }^{2}$ Present address: Helsinki Innovation Services, Tukholmankatu 8 A, 00290 Helsinki, Finland. ${ }^{3}$ Present address: MediSapiens Ltd, Erottajankatu 19B, 00130 Helsinki, Finland. ${ }^{4}$ Present address: Science for Life Laboratory, Department Oncology-Pathology, Karolinska Institutet, Tomtebodavägen 23, 17165 Solna, Sweden.

Received: 18 June 2015 Revised: 31 May 2016 Accepted: 15 June 2016 Published online: 04 July 2016

\section{References}

1. Chang M. Tamoxifen Resistance in Breast Cancer. Biomol Ther. 2012;20:256-67.

2. Riggins RB, Schrecengost RS, Guerrero MS, Bouton AH. Pathways to Tamoxifen Resistance. Cancer Lett. 2007;256:1-24.

3. Karnik PS, Kulkarni S, Liu XP, Budd GT, Bukowski RM. Estrogen receptor mutations in tamoxifen-resistant breast cancer. Cancer Res. 1994;54:349-53.

4. Toy W, Shen Y, Won H, Green B, Sakr RA, Will M, et al. ESR1 ligand-binding domain mutations in hormone-resistant breast cancer. Nat Genet. 2013;45:1439-45.

5. Robinson DR, Wu Y-M, Vats P, Su F, Lonigro RJ, Cao X, et al. Activating ESR1 mutations in hormone-resistant metastatic breast cancer. Nat Genet. 2013;45:1446-51.

6. Osborne CK, Bardou V, Hopp TA, Chamness GC, Hilsenbeck SG, Fuqua SAW, et al. Role of the estrogen receptor coactivator AIB1 (SRC-3) and HER-2/neu in tamoxifen resistance in breast cancer. J Natl Cancer Inst. 2003;95:353-61.

7. Ring A, Dowsett M. Mechanisms of tamoxifen resistance. Endocr Relat Cancer. 2004;11:643-58.

8. Gee JM, Robertson JF, Ellis IO, Nicholson RI. Phosphorylation of ERK1/2 mitogen-activated protein kinase is associated with poor response to anti-hormonal therapy and decreased patient survival in clinical breast cancer. Int J Cancer J Int Cancer. 2001;95:247-54

9. Ghayad SE, Vendrell JA, Ben Larbi S, Dumontet C, Bieche I, Cohen PA. Endocrine resistance associated with activated ErbB system in breast cancer cells is reversed by inhibiting MAPK or PI3K/Akt signaling pathways. Int J Cancer J Int Cancer. 2010;126:545-62.

10. Knowlden JM, Hutcheson IR, Jones HE, Madden T, Gee JMW, Harper ME, et al. Elevated levels of epidermal growth factor receptor/c-erbB2 heterodimers mediate an autocrine growth regulatory pathway in tamoxifen-resistant MCF-7 cells. Endocrinology. 2003;144:1032-44.

11. Zhou Y, Yau C, Gray JW, Chew K, Dairkee SH, Moore DH, et al. Enhanced NF kappa B and AP-1 transcriptional activity associated with antiestrogen resistant breast cancer. BMC Cancer. 2007:7:59

12. Ishii Y, Papa L, Bahadur U, Yue Z, Aguirre-Ghiso J, Shioda T, et al. Bortezomib enhances the efficacy of fulvestrant by amplifying the aggregation of the estrogen receptor, which leads to a proapoptotic unfolded protein response. Clin. Cancer Res. Off. J. Am. Assoc. Cancer Res. 2011;17:2292-300.

13. Trinh XB, Sas L, Van Laere SJ, Prové A, Deleu I, Rasschaert M, et al. A phase I study of the combination of endocrine treatment and bortezomib in patients with endocrine-resistant metastatic breast cancer. Oncol Rep. 2012;27:657-63.
14. Meijer D, van Agthoven T, Bosma PT, Nooter K, Dorssers LCJ. Functional screen for genes responsible for tamoxifen resistance in human breast cancer cells. Mol Cancer Res MCR. 2006;4:379-86.

15. Mendes-Pereira AM, Sims D, Dexter T, Fenwick K, Assiotis I, Kozarewa I, et al. Genome-wide functional screen identifies a compendium of genes affecting sensitivity to tamoxifen. Proc Natl Acad Sci U S A. 2012:109:2730-5.

16. Barone I, Brusco L, Gu G, Selever J, Beyer A, Covington KR, et al. Loss of Rho GDla and resistance to tamoxifen via effects on estrogen receptor a. J Natl Cancer Inst. 2011;103:538-52

17. Campbell RA, Bhat-Nakshatri P, Patel NM, Constantinidou D, Ali S, Nakshatri H. Phosphatidylinositol 3-kinase/AKT-mediated activation of estrogen receptor alpha: a new model for anti-estrogen resistance. J Biol Chem. 2001:276:9817-24

18. Holm C, Rayala S, Jirström K, Stål O, Kumar R, Landberg G. Association between Pak1 expression and subcellular localization and tamoxifen resistance in breast cancer patients. J Natl Cancer Inst. 2006;98:671-80.

19. Louie MC, McClellan A, Siewit C, Kawabata L. Estrogen receptor regulates E2F1 expression to mediate tamoxifen resistance. Mol Cancer Res MCR. 2010;8:343-52.

20. Block M, Gründker C, Fister S, Kubin J, Wilkens L, Mueller MD, et al. Inhibition of the AKT/mTOR and erbB pathways by gefitinib, perifosine and analogs of gonadotropin-releasing hormone I and II to overcome tamoxifen resistance in breast cancer cells. Int J Oncol. 2012;41:1845-54.

21. DeGraffenried LA, Friedrichs WE, Russell DH, Donzis EJ, Middleton AK, Silva $J M$, et al. Inhibition of mTOR activity restores tamoxifen response in breast cancer cells with aberrant Akt Activity. Clin Cancer Res Off J Am Assoc Cancer Res. 2004;10:8059-67.

22. Leary AF, Drury S, Detre S, Pancholi S, Lykkesfeldt AE, Martin L-A, et al. Lapatinib restores hormone sensitivity with differential effects on estrogen receptor signaling in cell models of human epidermal growth factor receptor 2-negative breast cancer with acquired endocrine resistance. Clin Cancer Res Off J Am Assoc Cancer Res. 2010;16:1486-97.

23. Aguilar H, Urruticoechea A, Halonen P, Kiyotani K, Mushiroda T, Barril X, et al. VAV3 mediates resistance to breast cancer endocrine therapy. Breast Cancer Res BCR. 2014;16:R53.

24. Jiang Z, Guo J, Shen J, Jin M, Xie S, Wang L. The role of estrogen receptor alpha in mediating chemoresistance in breast cancer cells. J Exp Clin Cancer Res CR. 2012:31:42.

25. Östling P, Leivonen S-K, Aakula A, Kohonen P, Mäkelä R, Hagman Z, et al. Systematic analysis of microRNAs targeting the androgen receptor in prostate cancer cells. Cancer Res. 2011;71:1956-67.

26. Pemovska T, Kontro M, Yadav B, Edgren H, Eldfors S, Szwajda A, et al. Individualized systems medicine strategy to tailor treatments for patients with chemorefractory acute myeloid leukemia. Cancer Discov. 2013:3:1416-29.

27. Koboldt DC, Zhang Q, Larson DE, Shen D, McLellan MD, Lin L, et al. VarScan 2: somatic mutation and copy number alteration discovery in cancer by exome sequencing. Genome Res. 2012;22:568-76.

28. Cingolani $\mathrm{P}$, Platts $\mathrm{A}$, Wang $\mathrm{LL}$, Coon $\mathrm{M}$, Nguyen $\mathrm{T}$, Wang $\mathrm{L}$, et al. A program for annotating and predicting the effects of single nucleotide polymorphisms, SnpEff: SNPs in the genome of Drosophila melanogaster strain w1118; iso-2; iso-3. Fly (Austin). 2012;6:80-92.

29. Fuentes Fajardo KV, Adams D. NISC Comparative Sequencing Program, Mason CE, Sincan M, Tifft C, et al. Detecting false-positive signals in exome sequencing. Hum Mutat. 2012;33:609-13.

30. Sulonen A-M, Ellonen P, Almusa H, Lepistö M, Eldfors S, Hannula S, et al Comparison of solution-based exome capture methods for next generation sequencing. Genome Biol. 2011;12:R94.

31. Seshan V, Olshen A. DNAcopy: DNA copy number data analysis. R package version 1.38.1. [Internet]. http://www.bioconductor.org/packages/release/ bioc/html/DNAcopy.html

32. Mermel CH, Schumacher SE, Hill B, Meyerson ML, Beroukhim R, Getz G GISTIC2.0 facilitates sensitive and confident localization of the targets of focal somatic copy-number alteration in human cancers. Genome Biol. 2011:12:R41.

33. Beroukhim R, Getz G, Nghiemphu L, Barretina J, Hsueh T, Linhart D, et al. Assessing the significance of chromosomal aberrations in cancer: methodology and application to glioma. Proc Natl Acad Sci U S A. 2007:104:20007-12.

34. Beroukhim R, Mermel CH, Porter D, Wei G, Raychaudhuri S, Donovan J, et al. The landscape of somatic copy-number alteration across human cancers. Nature. 2010;463:899-905. 
35. Yadav B, Pemovska T, Szwajda A, Kulesskiy E, Kontro M, Karjalainen R, Majumder MM, Malani D, Murumagi A, Knowles J, Porkka K, Heckman C, Kallioniemi O, Wennerberg K, Aittokallio T. Quantitative scoring of differential drug sensitivity for individually optimized anticancer therapies. Sci Rep. 2014;4.

36. Breitling R, Armengaud P, Amtmann A, Herzyk P. Rank products: a simple, yet powerful, new method to detect differentially regulated genes in replicated microarray experiments. FEBS Lett. 2004;573:83-92.

37. Subik K, Lee J-F, Baxter L, Strzepek T, Costello D, Crowley P, et al. The Expression Patterns of ER, PR, HER2, CK5/6, EGFR, Ki-67 and AR by Immunohistochemical Analysis in Breast Cancer Cell Lines. Breast Cancer Basic Clin Res. 2010;4:35-41.

38. Tang J, Szwajda A, Shakyawar S, Xu T, Hintsanen P, Wennerberg K, et al. Making sense of large-scale kinase inhibitor bioactivity data sets: a comparative and integrative analysis. J Chem Inf Model. 2014;54:735-43.

39. Krämer A, Green J, Pollard Jr J, Tugendreich S. Causal analysis approaches in Ingenuity Pathway Analysis. Bioinforma Oxf Engl. 2014;30:523-30.

40. Naundorf $H$, Becker M, Lykkesfeldt AE, Elbe B, Neumann C, Büttner B, et al. Development and characterization of a tamoxifen-resistant breast carcinoma xenograft. Br J Cancer. 2000;82:1844-50.

41. Pontiggia $O$, Rodriguez $V$, Fabris $V$, Raffo D, Bumaschny $V$, Fiszman $G$, et al. Establishment of an in vitro estrogen-dependent mouse mammary tumor model: a new tool to understand estrogen responsiveness and development of tamoxifen resistance in the context of stromal-epithelial interactions. Breast Cancer Res Treat. 2009;116:247-55.

42. Knowlden JM, Hutcheson IR, Barrow D, Gee JMW, Nicholson RI. Insulin-like growth factor-I receptor signaling in tamoxifen-resistant breast cancer: a supporting role to the epidermal growth factor receptor. Endocrinology. 2005;146:4609-18

43. Günthert AR, Gründker C, Olota A, Läsche J, Eicke N, Emons G. Analogs of $\mathrm{GnRH}-\mathrm{I}$ and $\mathrm{GnRH}-\mathrm{Il}$ inhibit epidermal growth factor-induced signal transduction and resensitize resistant human breast cancer cells to 4OH-tamoxifen. Eur J Endocrinol Eur Fed Endocr Soc. 2005;153:613-25.

44. Kurokawa $H$, Lenferink $A E$, Simpson JF, Pisacane PI, Sliwkowski MX, Forbes JT, et al. Inhibition of HER2/neu (erbB-2) and mitogen-activated protein kinases enhances tamoxifen action against HER2-overexpressing, tamoxifen-resistant breast cancer cells. Cancer Res. 2000;60:5887-94.

45. Creighton CJ, Massarweh S, Huang S, Tsimelzon A, Hilsenbeck SG, Osborne $\mathrm{CK}$, et al. Development of resistance to targeted therapies transforms the clinically associated molecular profile subtype of breast tumor xenografts. Cancer Res. 2008;68:7493-501.

46. Oh SJ, Kim O, Lee JS, Kim J-A, Kim MR, Choi HS, et al. Inhibition of angiogenesis by quercetin in tamoxifen-resistant breast cancer cells. Food Chem Toxicol Int J Publ Br Ind Biol Res Assoc. 2010:48:3227-34.

47. Xia W, Bisi J, Strum J, Liu L, Carrick K, Graham KM, et al. Regulation of survivin by ErbB2 signaling: therapeutic implications for ErbB2-overexpressing breast cancers. Cancer Res. 2006;66:1640-7.

48. van den Berg HW, Lynch M, Martin J, Nelson J, Dickson GR, Crockard AD. Characterisation of a tamoxifen-resistant variant of the ZR-75-1 human breast cancer cell line (ZR-75-9a1) and ability of the resistant phenotype. Br J Cancer. 1989;59:522-6.

49. Graham 2nd ML, Smith JA, Jewett PB, Horwitz KB. Heterogeneity of progesterone receptor content and remodeling by tamoxifen characterize subpopulations of cultured human breast cancer cells: analysis by quantitative dual parameter flow cytometry. Cancer Res. 1992;52:593-602.

50. Pink JJ, Jordan VC. Models of estrogen receptor regulation by estrogens and antiestrogens in breast cancer cell lines. Cancer Res. 1996;56:2321-30.

51. Cui Y, Parra I, Zhang M, Hilsenbeck SG, Tsimelzon A, Furukawa T, et al. Elevated expression of mitogen-activated protein kinase phosphatase 3 in breast tumors: a mechanism of tamoxifen resistance. Cancer Res. 2006;66:5950-9.

52. Holloway JN, Murthy S, El-Ashry D. A cytoplasmic substrate of mitogen-activated protein kinase is responsible for estrogen receptor-alpha down-regulation in breast cancer cells: the role of nuclear factor-kappaB. Mol Endocrinol Baltim Md. 2004;18:1396-410.

53. Chaikuad A, Tacconi EMC, Zimmer J, Liang Y, Gray NS, Tarsounas M, et al. A unique inhibitor binding site in ERK1/2 is associated with slow binding kinetics. Nat Chem Biol. 2014;10:853-60.

54. Reid G, Hübner MR, Métivier R, Brand H, Denger S, Manu D, et al. Cyclic, proteasome-mediated turnover of unliganded and liganded ERalpha on responsive promoters is an integral feature of estrogen signaling. Mol Cell. 2003:11:695-707.
55. Anbalagan M, Carrier L, Glodowski S, Hangauer D, Shan B, Rowan BG. KX-01, a novel Src kinase inhibitor directed toward the peptide substrate site, synergizes with tamoxifen in estrogen receptor a positive breast cancer. Breast Cancer Res Treat. 2012;132:391-409.

56. Cittelly DM, Das PM, Salvo VA, Fonseca JP, Burow ME, Jones FE. Oncogenic HER2\{Delta\}16 suppresses miR-15a/16 and deregulates BCL-2 to promote endocrine resistance of breast tumors. Carcinogenesis. 2010;31:2049-57.

57. Cannings E, Kirkegaard T, Tovey SM, Dunne B, Cooke TG, Bartlett JMS. Bad expression predicts outcome in patients treated with tamoxifen. Breast Cancer Res Treat. 2007;102:173-9.

58. van Agthoven T, Sieuwerts AM, Meijer D, Meijer-van Gelder ME, van Agthoven TLA, Sarwari R, et al. Selective recruitment of breast cancer anti-estrogen resistance genes and relevance for breast cancer progression and tamoxifen therapy response. Endocr Relat Cancer. 2010;17:215-30.

59. Musgrove EA, Sutherland RL. Biological determinants of endocrine resistance in breast cancer. Nat Rev Cancer. 2009;9:631-43.

60. Morrison G, Fu X, Shea M, Nanda S, Giuliano M, Wang T, et al. Therapeutic potential of the dual EGFR/HER2 inhibitor AZD8931 in circumventing endocrine resistance. Breast Cancer Res Treat. 2014;144:263-72.

61. Gee JMW, Harper ME, Hutcheson IR, Madden TA, Barrow D, Knowlden JM, et al. The antiepidermal growth factor receptor agent gefitinib (ZD1839/ Iressa) improves antihormone response and prevents development of resistance in breast cancer in vitro. Endocrinology. 2003;144:5105-17.

62. Arpino G, Gutierrez C, Weiss H, Rimawi M, Massarweh S, Bharwani L, et al. Treatment of human epidermal growth factor receptor 2-overexpressing breast cancer xenografts with multiagent HER-targeted therapy. J Nat Cancer Inst. 2007;99:694-705.

63. Cirillo F, Nassa G, Tarallo R, Stellato C, De Filippo MR, Ambrosino C, et al. Molecular mechanisms of selective estrogen receptor modulator activity in human breast cancer cells: identification of novel nuclear cofactors of antiestrogen-ERa complexes by interaction proteomics. J Proteome Res. 2013;12:421-31

64. Hong S-E, Kim E-K, Jin H-O, Kim H-A, Lee JK, Koh JS, et al. S6K1 inhibition enhances tamoxifen-induced cell death in MCF-7 cells through translational inhibition of Mcl-1 and survivin. Cell Biol Toxicol. 2013;29:273-82.

65. Winter GE, Radic B, Mayor-Ruiz C, Blomen VA, Trefzer C, Kandasamy RK, et al. The solute carrier SLC35F2 enables YM155-mediated DNA damage toxicity. Nat Chem Biol. 2014;10(9):768-73.

66. Brünner N, Boysen B, Jirus S, Skaar TC, Holst-Hansen C, Lippman J, et al. MCF7/LCC9: an antiestrogen-resistant MCF-7 variant in which acquired resistance to the steroidal antiestrogen $\mathrm{ICI} 182,780$ confers an early cross-resistance to the nonsteroidal antiestrogen tamoxifen. Cancer Res. 1997;57:3486-93.

67. Pisco AO, Brock A, Zhou J, Moor A, Mojtahedi M, Jackson D, et al. Non-Darwinian dynamics in therapy-induced cancer drug resistance. Nat Commun. 2013:4:2467.

68. Pisco AO, Huang S. Non-genetic cancer cell plasticity and therapy-induced stemness in tumour relapse: "What does not kill me strengthens me.". Br J Cancer. 2015;112:1725-32. 